\title{
A NON-STANDARD BOUNDARY VALUE PROBLEM RELATED TO GEOMAGNETISM
}

\author{
$\mathrm{BY}$ \\ RALF KAISER AND MICHAEL NEUDERT \\ Department of Mathematics, Universität Bayreuth, D-95440 Bayreuth, Germany
}

\begin{abstract}
Consider the following boundary value problem in the exterior space $\hat{S}^{d-1}=\left\{\mathbf{x} \in \mathbb{R}^{d}:|\mathbf{x}|>1\right\}$ of a sphere $S^{d-1}$ in two and three dimensions $(d=2,3)$ : Given a vector field $\mathbf{D}: S^{d-1} \rightarrow \mathbb{R}^{d}$ we ask for all harmonic vector fields $\mathbf{B}: \hat{S}^{d-1} \rightarrow \mathbb{R}^{d}$ which decay at least as fast as a dipole field at infinity and are parallel to $\mathbf{D}$ on $S^{d-1}$; i.e. there is $f: S^{d-1} \rightarrow \mathbb{R}$ such that $\mathbf{B}=f \mathbf{D}$. For $d=3$, this problem is related to the problem of reconstructing the geomagnetic field outside the earth from directional data measured on the earth's surface. The question for uniqueness or non-uniqueness is of particular interest here.

In this paper we characterize the solution space $V_{\mathbf{D}}^{\perp}$ of the boundary value problem as orthogonal complement of a certain set of functions determined by the vector field $\mathbf{D}$ in an appropriate Hilbert space. Based on the Hilbert space approach we determine $V_{\mathbf{D}}^{\perp}$ and its dimension $\operatorname{dim} V_{\mathbf{D}}^{\perp}$ for certain classes of vector fields $\mathbf{D}$. In particular, we find in $d=2$ for those fields $\mathbf{D}_{N}$ which are obtained by restricting a $2^{N}$-pole field on $S^{1}$, $\operatorname{dim}$ $V_{\mathbf{D}_{N}}^{\perp}=2(N-1)+1$. This result is robust in the sense that perturbations of $\mathbf{D}_{N}$ which are small in a certain norm do not change the dimension of the solution space. In $d=3$ we consider only the axisymmetric situation. Here, we find in the case that $\mathbf{D}$ is given by polynomials of order not larger than $N$ the upper bound $\operatorname{dim} V_{\mathbf{D}}^{\perp} \leq N$ and in the $2^{N}$-pole case $\operatorname{dim} V_{\mathbf{D}_{N}}^{\perp}=N$. For $N=1$ (dipole field) the result is proved to be robust, which implies uniqueness of the boundary value problem for all vector fields $\mathbf{D}$ close to $\mathbf{D}_{1}$. For $\mathbf{D}_{N}$ with $N \geq 2$ it is shown that uniqueness can be enforced if either the Hilbert space is truncated or if stronger decay conditions at infinity are imposed.
\end{abstract}

1. Introduction. It is a problem in the geophysical sciences to determine the magnetic field outside the earth if only the direction of the field is known on the earth's surface. The problem arises less in present day investigations, where complete measurements of the magnetic field vector (direction and intensity) are common, than in the interpretation of historical data sets: Before 1832, when Gauss invented a method of

Received May 9, 2002.

2000 Mathematics Subject Classification. Primary 35R25, 35R30, 86A25.

(C)2004 Brown University 
measuring absolute magnetic intensity, only declination (direction of the horizontal component) and inclination (the angle the field vector makes with the local horizontal) could be measured. The same problem arises in the interpretation of palaeomagnetic data records which provide directional information on a much simpler basis than information about intensity (see, e.g., Merrill \& McElhinny 1983).

Neglecting deviations from the spherical shape $S^{d-1}$ of the earth's surface and assuming the exterior region $\hat{S}^{d-1}$ to be insulating and to be free of sources of magnetic field, the above problem can be formalized as follows: Given a direction ficld $\mathbf{D} \in C^{0}\left(S^{d-1}, \mathbb{R}^{d}\right)$ we ask for all nontrivial vector fields $\mathbf{B} \in C^{1}\left(\hat{S}^{d-1}, \mathbb{R}^{d}\right)$ for which a scalar function $f: S^{d-1} \rightarrow \mathbb{R}$ exists such that the conditions

$$
\begin{array}{cc}
\nabla \times \mathbf{B}=0, \quad \nabla \cdot \mathbf{B}=0 & \text { in } \hat{S}^{d-1} \\
|\mathbf{B}(\mathbf{x})|=O\left(|\mathbf{x}|^{-d}\right) & \text { for }|\mathbf{x}| \rightarrow \infty \\
\mathbf{B}=f \mathbf{D} & \text { on } S^{d-1}
\end{array}
$$

are satisfied. The type of function $f$ which is appropriate here and the precise sense in which $(1.1)_{3}$ holds is specified in the next section. Let us just remark that $f$ need not obey a sign condition, a situation which is referred to by some anthors as "unsigned directional problem" (cf. Hulot et al. 1997). We will consider in the following the dimension $d=2$ and $d=3$. The case $d=2$ is much simpler to deal with; it will serve as a guide for the physical problem $d=3$.

The standard boundary value problems of potential theory specify either the normal component or the tangential components (with suitable consistency condition) on the boundary. If combined with an appropriate decay condition at infinity, existence and uniqueness of solutions is then guaranteed (cf., e.g., Kellogg 1967). No comparable results are known for problem (1.1). Considering the aforementioned applications, the focus of interest is less on specifying the most general direction ficld allowing for a solution of problem (1.1) than on specifying the amount of non-uniqueness in situations where a solution is already known to exist. Of course, due to the homogeneity of problem (1.1), uniqueness is always understood up to a multiplicative constant which remains free. The focus of the present paper is on this uniqueness problem, too.

Problem (1.1) is only one in a whole series of similar inverse problems in geophysics, all concerned with recovering the magnetic field (or other fields) from incomplete or illposed data. A problem, complementary to (1.1), for example, tries to determine the field $\mathbf{B}$ in $\hat{S}^{d-1}$ from total intensity data on $S^{d-1}$ (cf. Backus 1968, 1970). This problem has been prompted by the fact that total intensity can typically more easily and more cheaply be measured than directions. Early satellite measurements, for example, provide only intensity data. Another problem consists in inferring motions of the conducting fluid core of the earth from secular variations of the magnetic field (cf. Hide 1986, Bloxham \& Jackson 1991). In highly-conducting fluids, the magnetic field is "frozen" into the fluid and core motions should clearly have an influence on the geomagnetic secular variation. Obviously, in all of these problems, the key question concerns uniqueness or non-uniqueness of solutions.

Several authors have already dealt with problem (1.1) from a geophysical perspective and have, in particular, tried to answer the uniqueness problem. Kono (1976) claimed 
to have proved uniqueness of the solution of problem (1.1) for $d=3$ under an even weaker condition than $(1.1)_{2}$. The proof, however, turned out to be erroneous (Hulot et al. (1997) indicate some loopholes in Kono's proof) and the claim has been disproved by Proctor \& Gubbins (1990) who constructed a counterexample. These authors considered the 2-dimensional as well as the 3-dimensional problem. In two dimensions, they discuss the problem in the framework of the theory of complex variables and establish, e.g., a relation between the rotation number of $\mathbf{D}$ on $S^{1}$, the rotation number of $\mathbf{B}$ at infinity, and the number of zeros of $\mathbf{B}$ in $\hat{S}_{1}$. This relation suggests an upper bound on the number of free parameters in the solution equal to $2(r-2)$ where $r$ is the rotation number of $\mathbf{D}$ on $S^{1}$. This would imply uniqueness for dipole-type direction fields $(r=$ 2 ). However, no rigorous statement concerning the dimension of the solution space of problem (1.1) is made and the discussion remains vague. In three dimensions Proctor \& Gubbins proceed by example. By numerical means, they produce three different solutions for an octupole-type direction field $(r=4)$. This clearly establishes nonuniqueness in the general case. Moreover, the authors conjecture that uniqueness can be enforced if solutions are represented by truncated spherical harmonic expansions with fixed truncation level.

A recent paper dealing with the uniqueness problem in three dimensions is that of Hulot et al. $(1997)^{1}$ : In the framework of potential theory, the authors derive an upper bound on the dimension of the solution space equal to $l-1$ with $l$ being the number of loci on $S^{2}$ (in fact the surface need not be spherical) where the tangential component of the direction field vanishes. The result is based on a beautiful application of Hopf's maximum principle applied to the potential of $\mathbf{B}$. Note that the regularity condition used by Hulot et al. has to be understood in the sense of Courant \& Hilbert (1962), which implies some condition at infinity. In fact, in order to ensure uniqueness in unbounded domains, one needs either uniform decay to zero at infinity or at least a Phragmèn-Lindelöftype argument which requires some growth restriction at infinity (see, e.g., Protter \& Weinberger 1984). In any case, this upper bound implies uniqueness for direction fields with no more than two poles.

In contrast to these results, our approach allows us to determine the precise dimension of the solution space and to construct the corresponding solutions at least for certain classes of direction fields. For this purpose the space of functions $f$ in $(1.1)_{3}$ is characterized in Sec. two as orthogonal complement in $L^{2}\left(S^{d-1}\right)$ of a set of functions determined by the direction field $\mathbf{D}$. Special fields $\mathbf{D}_{N}$ obtained by restricting $2^{N}$-pole fields on $S^{d-1}$ turn out to be of particular importance. The 2 -dimensional case, which is much simpler than $d=3$, is treated in Sec. 3: The solution space can explicitly be determined and we obtain for its dimension $\operatorname{dim} V_{\mathbf{D}_{N}}^{\perp}=2(N-1)+1$. The fields $\mathbf{D}_{N}$ are special in that they have a constant rotation rate $N$ which relates to the rotation number $r$ by $r=N+1$. Using a Paley-Wiener-type expansion theorem, we prove that for fields $\mathbf{D}$ with nonconstant rotation rate, $\operatorname{dim} V_{\mathbf{D}_{N}}^{\perp}=2(N-1)+1$ with $N$ being the mean rotation rate, provided that the deviations of the rotation rate from $N$ are small in a norm which involves the derivative of the rotation rate.

\footnotetext{
${ }^{1}$ This paper came to our attention only when our work was already completed.
} 
In three dimensions we treat only the axisymmetric situation (Sec. 4): If $\mathbf{D}$ is given by polynomials of order not larger than $N$ we derive the upper bound $\operatorname{dim} V_{\mathbf{D}}^{\perp} \leq N$ and in the multipole case we find $\operatorname{dim} V_{\mathbf{D}_{N}}^{\perp}=N$. The solutions are given in the form of recursively defined series whose convergence is the crucial point to prove. Here, we make use of the so-called Clebsch-Gordan coefficients, which appear in the decomposition of tensor representations of the rotation group in its irreducible components, and apply a theorem due to Eneström and Kakeya bounding the zeros of certain polynomials. Furthermore, uniqueness is proved to hold for all direction fields which are close to $\mathbf{D}_{1}$ in a higher-derivative norm. Some useful formulas about Legendre polynomials, spherical harmonics, and Clebsch-Gordan coefficients that are needed in the preceeding proofs are collected in two appendices.

Finally, the question is treated whether in case of non-uniqueness additional conditions can enforce uniqueness. Proctor \& Gubbins (1990) conjecture the finite-dimensional representation of the solutions by spherical harmonics as such a condition. We answer this question for the direction fields $\mathbf{D}_{N}, N \geq 2$ and find in $d=3$ uniqueness but not in $d=2$. Another possibility is to require stronger decay conditions at infinity. In fact, replacing condition $(1.1)_{2}$ by $|\mathbf{B}|=O\left(|\mathbf{x}|^{-(d+N-1)}\right)$ for $|\mathbf{x}| \rightarrow \infty$ implies uniqueness for the direction fields $\mathbf{D}_{N}$ in two as well as three dimensions.

A conjecture about the relation between the dimension of the solution space and a suitably defined rotation number for axisymmetric 3 -dimensional direction fields concludes the paper.

2. Hilbert space approach. This section reformulates problem (1.1) as a Hilbert space problem with Hilbert space $\mathcal{H}=L^{2}\left(S^{d-1}\right)$. For this purpose, we introduce a potential for the harmonic field $\mathbf{B}$ and explain the boundary values of $\mathbf{B}$ in the sense of traces. The unique solvability of the Neumann problem with such generalized boundary values is proved in Lemma 2.1. The proper Hilbert space criteria are then formulated separately for $d=2$ and $d=3$ (Theorems 2.2 and 2.3 ); the $3 \mathrm{~d}$-axisymmetric case is formulated in Corollary 2.4.

Let us begin with some notation:

$$
S^{d-1}:=\left\{\mathbf{x} \in \mathbb{R}^{d}:|\mathbf{x}|=1\right\}, \quad \hat{S}^{d-1}:=\left\{\mathbf{x} \in \mathbb{R}^{d}:|\mathbf{x}|>1\right\} .
$$

In the following we will make use of polar coordinates $(r, \varphi)$ in $\mathbb{R}^{2}$ defining a coordinate system $\left\{\mathbf{e}_{r}, \mathbf{e}_{\varphi}\right\}$ as well as of spherical coordinates $(r, \theta, \varphi)$ in $\mathbb{R}^{3}$ with $\theta \in[0, \pi], \varphi \in$ $[0,2 \pi]$, and the coordinate system $\left\{\mathbf{e}_{r}, \mathbf{e}_{\theta}, \mathbf{e}_{\varphi}\right\}$. Gradients then take the form

$$
\begin{aligned}
& \nabla \Psi=\partial_{r} \Psi \mathbf{e}_{r}+\frac{1}{r} \partial_{\varphi} \Psi \mathbf{e}_{\varphi}, \quad d=2, \\
& \nabla \Psi=\partial_{r} \Psi \mathbf{e}_{r}+\frac{1}{r} \partial_{\theta} \Psi \mathbf{e}_{\theta}+\frac{1}{r \sin \theta} \partial_{\varphi} \Psi \mathbf{e}_{\varphi}, \quad d=3 .
\end{aligned}
$$

The volume element $d \Omega$ on $S^{d-1}$ is $d \varphi$ for $d=2$ and $\sin \theta d \theta d \varphi$ for $d=3$. In order to simplify the notation, a function $f: S^{d-1} \rightarrow \mathbb{C}(\mathbb{R})$ and its representation in polar or spherical coordinates will be denoted by the same symbol. 
As Hilbert space we use

$$
\mathcal{H}:=L^{2}\left(S^{d-1}\right)=\left\{h:\left.S^{d-1} \rightarrow \mathbb{C}\left|\int_{S^{d-1}}\right| h\right|^{2} d \Omega<\infty\right\}
$$

with scalar product

$$
\left(h_{1}, h_{2}\right)_{\mathcal{H}}:=\int_{S^{d-1}} h_{1} h_{2}^{*} d \Omega
$$

and norm $\|h\|:=\sqrt{(h, h)_{\mathcal{H}}}$. "* " means complex conjugation.

With the definitions

$$
Y_{0,2}^{0}:=\frac{1}{\sqrt{2 \pi}}, \quad Y_{n, 2}^{1}:=\frac{1}{\sqrt{\pi}} \sin n \varphi, \quad Y_{n, 2}^{-1}:=\frac{1}{\sqrt{\pi}} \cos n \varphi, \quad Y_{n, 3}^{k}:=Y_{n}^{k}(\theta, \varphi),
$$

where $Y_{n}^{k}(\theta, \varphi)$ denote the usual spherical harmonics (cf. Appendix A), the set of eigenfunctions of the Laplace--Beltrami operator can be described uniformly for $d=2$ and $d=3$ by

$$
\left\{Y_{n, d}^{k}: n \in \mathbb{N}_{0}, k \in K(n, d)\right\},
$$

where $K(0, d)=\{0\}, K(n, 2)=\{-1,+1\}, K(n, 3)=\{-n, \ldots,+n\}, n \in \mathbb{N}$. The set $(2.1)$ forms a complete orthonormal system in $L^{2}\left(S^{d-1}\right)$ and allows the representation

$$
\Psi=\sum_{n=0}^{\infty} \sum_{k \in K(n, d)} a_{n k} r^{-(n+d-2)} Y_{n, d}^{k}
$$

of every harmonic function $\Psi$ in $\hat{S}^{d-1}$ vanishing at infinity.

In the following, we will prescribe boundary values which are only supposed to be elements of $L^{2}\left(S^{d-1}\right)$. Let us define for this purpose for any smooth function $\Psi$ in $\hat{S}^{d-1}$ and for $r>1:^{2}$

$$
\left.\partial_{r} \Psi(r .)\right|_{S^{d-1}}: \quad S^{d-1} \rightarrow \mathbb{C}, \quad \hat{\mathbf{x}} \mapsto \partial_{r} \Psi(r \hat{\mathbf{x}}):=\nabla \Psi(r \hat{\mathbf{x}}) \cdot \hat{\mathbf{x}}
$$

A suitable Neumann boundary value problem for Laplace's equation now reads:

Let $\gamma \in L^{2}\left(S^{d-1}\right)$ with $\int_{S^{d-1}} \gamma d \Omega=0$. Determine all functions $\Psi \in C^{2}\left(\hat{S}^{d-1}, \mathbb{C}\right)$ satisfying

$$
\begin{aligned}
\Delta \Psi & =0 & & \text { in } \hat{S}^{d-1} \\
|\Psi(\mathbf{x})| & =O\left(|\mathbf{x}|^{-(d-1)}\right), & & \text { for }|\mathbf{x}| \rightarrow \infty, \\
\left\|\left.\partial_{r} \Psi(r .)\right|_{S^{d-1}}-\gamma\right\| & \rightarrow 0, & & r \searrow 1 .
\end{aligned}
$$

Here, $r \searrow 1$ means $r \rightarrow 1$ where $r>1$.

LEMma 2.1. Problem (2.4) is uniquely solvable.

Proof. (i) Let $\gamma=\sum_{n=1}^{\infty} \sum_{k \in K(n, d)} b_{n k} Y_{n, d}^{k}$ with $\left(b_{n k}\right)_{n k} \subset \mathbb{C}$. Set

$$
\Psi:=-\sum_{n=1}^{\infty} \sum_{k \in K(n, d)} \frac{b_{n k}}{n+d-2} r^{-(n+d-2)} Y_{n, d}^{k}
$$

\footnotetext{
${ }^{2}$ In the following, $\hat{\mathbf{x}}$ denote unit vectors.
} 
with the series converging uniformly for $r>r_{0}>1$ and thus $\Psi$ being a harmonic function in $\hat{S}^{d-1}$, satisfying $(2.4)_{2}$. Now assume $\epsilon>0$ and choose $N_{\epsilon} \in \mathbb{N}$ such that

$$
\left\|\sum_{n=N_{\epsilon}+1}^{\infty} \sum_{k \in K(n . d)} b_{n k} Y_{n, d}^{k}\right\|^{2}=\sum_{n=N_{\epsilon}+1}^{\infty} \sum_{k \in K(n, d)}\left|b_{n k}\right|^{2}<\frac{\epsilon}{2} .
$$

Thus, for $r \geq 1$,

$$
\left\|\sum_{n=N_{\epsilon}+1}^{\infty} \sum_{k \in K(n, d)} b_{n k}\left(1-r^{-(n+d-1)}\right) Y_{n, d}^{k}\right\|^{2}<\frac{\epsilon}{2} .
$$

Now assume $\delta_{\epsilon}>0$ such that

$$
\forall r \in\left[1,1+\delta_{\epsilon}\right): \quad\left|1-r^{-\left(N_{\epsilon}+d-1\right)}\right|^{2} \sum_{n=1}^{N_{\epsilon}} \sum_{k \in K(n . d)}\left|b_{n k}\right|^{2}<\frac{\epsilon}{2} .
$$

Then for $r \in\left[1,1+\delta_{\epsilon}\right)$, we also have

$$
\left\|\sum_{n=1}^{N_{\epsilon}} \sum_{k \in K(n, d)} b_{n k}\left(1-r^{-(n+d-1)}\right) Y_{n, d}^{k}\right\|^{2}<\frac{\epsilon}{2}
$$

and (2.6) and (2.7) imply $\left\|\left.\partial_{r} \Psi(r)\right|_{.S^{d-1}}-\gamma\right\| \rightarrow 0, r \searrow 1$.

(ii) Let $\Psi=\sum_{n=1}^{\infty} \sum_{k \in K(n, d)} a_{n k} r^{-(n+d-2)} Y_{n, d}^{k}$ be any harmonic function in $\hat{S}^{d-1}$ satisfying $\left\|\left.\partial_{r} \Psi(r)\right|_{.S^{d-1}}\right\| \rightarrow 0, r \searrow 1$. Then, for $\hat{\mathbf{x}} \in S^{d-1}$ and $r>1$,

$$
\partial_{r} \Psi(r \hat{\mathbf{x}})=-\sum_{n=1}^{\infty} \sum_{k \in K(n, d)} a_{n k}(n+d-2) r^{-(n+d-1)} Y_{n . d}^{k}(\hat{\mathbf{x}})
$$

and

thus $\Psi=0$.

$$
a_{n k}=\frac{1}{n+d-2} \lim _{r \searrow 1}\left(\left.\partial_{r} \Psi(r .)\right|_{S^{d-1}}, Y_{n, d}^{k}\right)_{\mathcal{H}}=0
$$

The analogue of (2.3) for the other components is:

$$
\begin{array}{ll}
\left.\partial_{\varphi} \Psi(r .)\right|_{S^{1}}: & S^{1} \rightarrow \mathbb{C}, \quad \hat{\mathbf{x}} \mapsto \partial_{\varphi} \Psi(r \hat{\mathbf{x}}):=r \nabla \Psi(r \hat{\mathbf{x}}) \cdot \mathbf{e}_{\varphi}(r \hat{\mathbf{x}}), \\
\left.\partial_{\theta} \Psi(r .)\right|_{S^{2}}: & S^{2} \rightarrow \mathbb{C}, \quad \hat{\mathbf{x}} \mapsto \partial_{\theta} \Psi(r \hat{\mathbf{x}}):=r \nabla \Psi(r \hat{\mathbf{x}}) \cdot \mathbf{e}_{\theta}(r \hat{\mathbf{x}}), \\
\left.\partial_{\varphi} \Psi(r .)\right|_{S^{2}}: & S^{2} \rightarrow \mathbb{C}, \quad \hat{\mathbf{x}} \mapsto \partial_{\varphi} \Psi(r \hat{\mathbf{x}}):=r \sin \theta(r \hat{\mathbf{x}}) \nabla \Psi(r \hat{\mathbf{x}}) \cdot \mathbf{e}_{\varphi}(r \hat{\mathbf{x}}) .
\end{array}
$$

We are now in the position to give problem (1.1) a more precise formulation. Using the potential (2.2) for $\mathbf{B}$ and the generalized boundary values (2.3), (2.8) $)_{1}$, we define in two dimensions:

Problem $\mathrm{P}_{2}$. Let $\mathbf{D}=D_{r}(\varphi) \mathbf{e}_{r}+D_{\varphi}(\varphi) \mathbf{e}_{\varphi} \in C^{0}\left(S^{1}, \mathbb{R}^{2}\right)$. Determine all functions $f \in \mathcal{H}=L^{2}\left(S^{1}\right)$ such that the following boundary value problem is solvable, i.e., there is $\Psi \in C^{2}\left(\hat{S}^{1}, \mathbb{R}\right)$ satisfying the conditions

$$
\begin{aligned}
\Delta \Psi & =0 & & \text { in } \hat{S}^{1}, \\
|\Psi(\mathbf{x})| & =O\left(|\mathbf{x}|^{-1}\right) & & \text { for }|\mathbf{x}| \rightarrow \infty, \\
\nabla \Psi & =f \mathbf{D} & & \text { on } S^{1} .
\end{aligned}
$$


The boundary condition $(2.9)_{3}$ holds in the sense of

$$
\begin{gathered}
\left\|\left.\partial_{r} \Psi(r .)\right|_{S^{1}}-f D_{r}\right\| \rightarrow 0 \quad \text { for } r \searrow 1 \\
\left\|\left.\partial_{\varphi} \Psi(r .)\right|_{S^{1}}-f D_{\varphi}\right\| \rightarrow 0 \quad \text { for } r \searrow 1 .
\end{gathered}
$$

In three dimensions it is convenient to allow for complex potentials and, consequently, complex functions $f$. For real direction fields $\mathbf{D}$, real and imaginary parts of $\Psi$ (and $f$ ) are then separately solutions and can be identified with physical quantities. We define:

Problem $\mathrm{P}_{3}$. Let $\mathbf{D}=D_{r}(\theta, \varphi) \mathbf{e}_{r}+D_{\theta}(\theta, \varphi) \mathbf{e}_{\theta}+D_{\varphi}(\theta, \varphi) \mathbf{e}_{\varphi} \in C^{0}\left(S^{2}, \mathbb{R}^{3}\right)$. Determine all functions $f \in \mathcal{H}=L^{2}\left(S^{2}\right)$ such that the following boundary value problem is solvable, i.e., there is $\Psi \in C^{2}\left(\hat{S}^{2}, \mathbb{C}\right)$ satisfying the conditions:

$$
\begin{aligned}
\Delta \Psi & =0 & & \text { in } \hat{S}^{2}, \\
|\Psi(\mathbf{x})| & =O\left(|\mathbf{x}|^{-2}\right) & & \text { for }|\mathbf{x}| \rightarrow \infty \\
\nabla \Psi & =f \mathbf{D} & & \text { on } S^{2} .
\end{aligned}
$$

The boundary condition $(2.11)_{3}$ holds in the sense of

$$
\begin{aligned}
\left\|\left.\partial_{r} \Psi(r .)\right|_{S^{2}}-f D_{r}\right\| \rightarrow 0 & \text { for } r \searrow 1, \\
\left\|\left.\partial_{\theta} \Psi(r .)\right|_{S^{2}} \sin \theta-f D_{\theta} \sin \theta\right\| \rightarrow 0 & \text { for } r \searrow 1, \\
\left\|\left.\partial_{\varphi} \Psi(r .)\right|_{S^{2}}-f D_{\varphi} \sin \theta\right\| \rightarrow 0 & \text { for } r \searrow 1 .
\end{aligned}
$$

Remark 2.2. Due to Lemma 2.1, problem $\mathrm{P}_{d}$ is equivalent to problem (1.1) if the direction field $\mathbf{D}$ is assumed to be $\neq 0$ almost everywhere on $S^{d-1}$ : For a solution $f \in \mathcal{H}$ of $\mathrm{P}_{d}$, the corresponding potential $\Psi$ is uniquely determined by

$$
\Psi=\sum_{n=1}^{\infty} \sum_{k \in K(n, d)} a_{n k} r^{-(n+d-2)} Y_{n, d}^{k}
$$

with

$$
a_{n k}=-\frac{1}{n+d-2} \lim _{r \searrow 1}\left(\left.\partial_{r} \Psi(r .)\right|_{S^{d-1}}, Y_{n, d}^{k}\right)_{\mathcal{H}} .
$$

Conversely, given a potential $\Psi$ and a direction field $\mathbf{D}$ on $S^{d-1}$ with $\mathbf{D} \neq 0$ almost everywhere, there is at most one $f \in \mathcal{H}$ such that the boundary conditions (2.10) or (2.12) are fulfilled.

Problem $\mathrm{P}_{2}$ allows now the following equivalent characterization:

Theorem 2.3. Let $\mathbf{D}=D_{r}(\varphi) \mathbf{e}_{r}+D_{\varphi}(\varphi) \mathbf{e}_{\varphi} \in C^{0}\left(S^{1}, \mathbb{R}^{2}\right)$ and $f \in L^{2}\left(S^{1}\right)$. Moreover, let

$$
\begin{aligned}
& T_{n}:=D_{r}(\varphi) \sin n \varphi+D_{\varphi}(\varphi) \cos n \varphi \\
& S_{n}:=D_{r}(\varphi) \cos n \varphi-D_{\varphi}(\varphi) \sin n \varphi
\end{aligned} \quad n \in \mathbb{N}_{0},
$$

and

$$
V_{\mathbf{D}}:=\overline{\operatorname{Span}\left\{T_{n}, S_{n}: n \in \mathbb{N}_{0}\right\}}
$$

Then, $f$ solves Problem $\mathrm{P}_{2}$ if and only if $f \in V_{\mathbf{D}}^{\perp}$, i.e., $\forall n \in \mathbb{N}_{0}$ :

$$
\int_{S^{1}} f T_{n} d \Omega=\int_{S^{1}} f S_{n} d \Omega=0 .
$$


Proof. (i) Let $f$ be a solution of $\mathrm{P}_{2}$ and let

$$
\Psi=\sum_{n=1}^{\infty} \frac{1}{r^{n}}\left(a_{n} \cos n \varphi+b_{n} \sin n \varphi\right)
$$

be the corresponding solution of (2.9). We have

$$
\begin{aligned}
& \partial_{r} \Psi=-\sum_{n=1}^{\infty} \frac{n}{r^{n+1}}\left(a_{n} \cos n \varphi+b_{n} \sin n \varphi\right), \\
& \partial_{\varphi} \Psi=-\sum_{n=1}^{\infty} \frac{n}{r^{n}}\left(a_{n} \sin n \varphi-b_{n} \cos n \varphi\right)
\end{aligned}
$$

with the right-hand sides uniformly converging in $\hat{S}^{1}$. With $D_{r}, D_{\varphi} \in C^{0}\left(S^{1}, \mathbb{R}^{2}\right)$ and $f \in L^{2}\left(S^{1}\right)$, we also have $f D_{r}, f D_{\varphi} \in L^{2}\left(S^{1}\right)$ and obtain from (2.10) and (2.15) for $n \in \mathbb{N}_{0}$ (and with $\left.a_{0}:=0, b_{0}:=0\right)$

$$
\begin{aligned}
\int_{-\pi}^{\pi} f(\varphi) D_{r}(\varphi) \cos n \varphi d \varphi & =\lim _{r \searrow 1} \int_{S^{1}} \partial_{r} \Psi(r .) \cos n \varphi d \Omega \\
& =\lim _{r \searrow 1} \int_{-\pi}^{\pi} \partial_{r} \Psi\left(r \mathbf{e}_{r}\right) \cos n \varphi d \varphi=-n \pi a_{n}
\end{aligned}
$$

and analogously

$$
\begin{gathered}
\int_{-\pi}^{\pi} f(\varphi) D_{r}(\varphi) \sin n \varphi d \varphi=-n \pi b_{n} \\
\int_{-\pi}^{\pi} f(\varphi) D_{\varphi}(\varphi) \cos n \varphi d \varphi=n \pi b_{n}, \quad \int_{-\pi}^{\pi} f(\varphi) D_{\varphi}(\varphi) \sin n \varphi d \varphi=-n \pi a_{n} .
\end{gathered}
$$

Eliminating $a_{n}$ and $b_{n}$ then yields the conditions (2.13).

(ii) Let $f \in V_{\mathrm{D}}^{\perp}$. Define $\gamma:=f D_{r}$ and let $\Psi$ be the unique solution of problem (2.4) according to Lemma 2.1. It remains to prove $(2.10)_{2}$.

Let

$$
\gamma=f D_{r}=\sum_{n=1}^{\infty}\left(a_{n} \cos n \varphi+b_{n} \sin n \varphi\right)
$$

Then we have

$$
\sum_{n=1}^{\infty}\left(\left|a_{n}\right|^{2}+\left|b_{n}\right|^{2}\right)=\pi\|\gamma\|^{2}
$$

From (2.13) and (2.18) we obtain

$$
\int_{S^{1}} f D_{\varphi} \cos n \varphi d \Omega=-\pi b_{n}, \quad \int_{S^{1}} f D_{\varphi} \sin n \varphi d \Omega=\pi a_{n}
$$

and thus

$$
f D_{\varphi}=\sum_{n=1}^{\infty}\left(a_{n} \sin n \varphi-b_{n} \cos n \varphi\right)
$$


Equations (2.18) and (2.5) in the proof of Lemma 2.1 yield

$$
\Psi\left(r \mathbf{e}_{r}\right)=-\sum_{n=1}^{\infty} \frac{1}{n r^{n}}\left(a_{n} \cos n \varphi+b_{n} \sin n \varphi\right)
$$

and therefore

$$
\partial_{\varphi} \Psi\left(r \mathbf{e}_{r}\right)=-\sum_{n=1}^{\infty} \frac{1}{r^{n}}\left(a_{n} \sin n \varphi-b_{n} \cos n \varphi\right)
$$

where, because of (2.19), the series on the right-hand side converges uniformly in $\hat{S}^{1}$. Comparing (2.21) in the limit $r \searrow 1$ with (2.20) furnishes $(2.10)_{2}$.

The corresponding result for $\mathrm{P}_{3}$ reads:

Theorem 2.4. Let $\mathbf{D} \in C^{0}\left(S^{2}, \mathbb{R}^{3}\right)$ as in Problem $\mathrm{P}_{3}$ and $f \in \mathcal{H}=L^{2}\left(S^{2}\right)$. Moreover, let for $n \in \mathbb{N}, k \in\{-n, \ldots,+n\}$

$$
\begin{aligned}
T_{-1}^{0} & :=D_{r} \\
T_{0}^{0} & :=D_{\theta} \sin \theta Y_{0}^{0}-\frac{1}{\sqrt{3}} D_{r} Y_{1}^{0}, \\
T_{n}^{k} & :=D_{\theta} \sin \theta Y_{n}^{k}+D_{r}\left(\frac{n-1}{n} \sqrt{\frac{n^{2}-k^{2}}{4 n^{2}-1}} Y_{n-1}^{k}-\sqrt{\frac{(n+1)^{2}-k^{2}}{4(n+1)^{2}-1}} Y_{n+1}^{k}\right), \\
S_{0}^{0} & :=D_{\varphi} \sin \theta Y_{0}^{0}, \\
S_{n}^{k} & :=\left(-i k D_{r}+(n+1) D_{\varphi} \sin \theta\right) Y_{n}^{k},
\end{aligned}
$$

where $Y_{n}^{k}:=Y_{n}^{k}(\theta, \varphi)$, and

$$
V_{\mathbf{D}}:=\overline{\operatorname{Span}\left\{T_{-1}^{0}, T_{n}^{k}, S_{n}^{k}: n \in \mathbb{N}_{0}, k=-n, \ldots, n\right\}}
$$

Then $f$ is a solution of Problem $\mathrm{P}_{3}$ if and only if $f \in V_{\mathbf{D}}^{\perp}$, i.e., $\forall n \in \mathbb{N}_{0}, k=-n, \ldots, n$ :

$$
\int_{S^{2}} f{T_{-1}^{0}}^{*} d \Omega=\int_{S^{2}} f T_{n}^{k^{*}} d \Omega=\int_{S^{2}} f S_{n}^{k^{*}} d \Omega=0 .
$$

Proof. (i) Let $f$ be a solution of $\mathrm{P}_{3}$ and let

$$
\Psi:=\sum_{n \in \mathbb{N}} \sum_{k=-n}^{+n} c_{n k} r^{-(n+1)} Y_{n}^{k}
$$

be the corresponding solution of the boundary value problem (2.11). With (A.9), the series

$$
\sum_{n \in \mathbb{N}} \sum_{k=-n}^{+n}(n+1) c_{n k} r^{-(n+2)} Y_{n}^{k}, \quad \sum_{n \in \mathbb{N}} \sum_{k=-n}^{+n} c_{n k} r^{-(n+2)} \partial_{\theta} Y_{n}^{k}
$$


are uniformly converging in every compact subset of $\left\{x \in \mathbb{R}^{3}: r>1,0<\theta<\pi\right\}$. Thus, using the abbreviation $N_{n}^{k}$ in (A.9), we obtain

$$
\begin{aligned}
\partial_{r} \Psi & =-\sum_{n=1}^{\infty} \sum_{k=-n}^{n}(n+1) c_{n k} r^{-(n+2)} Y_{n}^{k}, \\
\sin \theta \partial_{\theta} \Psi & =\sum_{n=1}^{\infty} \sum_{k=-n}^{n} c_{n k} r^{-(n+1)}\left(n N_{n+1}^{k} Y_{n+1}^{k}-(n+1) N_{n}^{k} Y_{n-1}^{k}\right) \\
& =\sum_{n=0}^{\infty} \sum_{k=-n}^{n}\left(c_{n-1, k} \frac{(n-1) N_{n}^{k}}{r^{n}}-c_{n+1, k} \frac{(n+2) N_{n+1}^{k}}{r^{n+2}}\right) Y_{n}^{k}, \\
\partial_{\varphi} \Psi & =\sum_{n=1}^{\infty} \sum_{k=-n}^{n} i k c_{n k} r^{-(n+1)} Y_{n}^{k} .
\end{aligned}
$$

Since $D_{r}, D_{\theta}, D_{\varphi} \in C^{0}\left(S^{2}, \mathbb{R}\right)$ and $f \in L^{2}\left(S^{2}\right)$, we also have $f D_{r}, f D_{\theta} \sin \theta, f D_{\varphi} \sin \theta \in$ $L^{2}\left(S^{2}\right)$ and from (2.12) and (2.23) we conclude for $n \in \mathbb{N}_{0}, k=-n, \ldots, n$ (with $c_{-1 . k}:=0$ )

$$
\begin{aligned}
\int_{S^{2}} f D_{r} Y_{n}^{k^{*}} d \Omega & =\lim _{r \searrow 1} \int_{S^{2}} \partial_{r} \Psi(r .) Y_{n}^{k^{*}} d \Omega=-(n+1) c_{n k} \\
\int_{S^{2}} f D_{\theta} \sin \theta Y_{n}^{k^{*}} d \Omega & =\lim _{r \searrow 1} \int_{S^{2}} \sin \theta \partial_{\theta} \Psi(r .) Y_{n}^{k^{*}} d \Omega \\
& =c_{n-1, k}(n-1) N_{n}^{k}-c_{n+1, k}(n+2) N_{n+1}^{k} \\
\int_{S^{2}} f D_{\varphi} \sin \theta Y_{n}^{k^{*}} d \Omega & =\lim _{r \searrow 1} \int_{S^{2}} \partial_{\varphi} \Psi(r .) Y_{n}^{k^{*}} d \Omega=i k c_{n k}
\end{aligned}
$$

From $(2.11)_{2}$ follows $c_{00}=0$ and thus, using $(2.24)_{1}$ and $(2.24)_{2}$,

$$
\int_{S^{2}} f D_{r} d \Omega=0, \quad \int_{S^{2}}\left(D_{\theta} \sin \theta Y_{0}^{0^{*}}-\frac{1}{\sqrt{3}} D_{r} Y_{0}^{1^{*}}\right) f d \Omega=0 .
$$

$(2.24)_{1},(2.24)_{2}$ and $(2.24)_{1},(2.24)_{3}$, respectively, yield the rest of $(2.22)$.

(ii) Now let $f \in L^{2}\left(S^{2}\right)$ satisfy the conditions (2.22). Set $\gamma:=f D_{r}$ and let $\Psi$ be the unique solution (2.5) of problem (2.4) according to Lemma 2.1. Set $c_{n k}:=-b_{n k} /(n+1)$. Then $\sum_{n, k}\left|(n+1) c_{n k}\right|^{2}=\sum_{n, k}\left|b_{n k}\right|^{2}<\infty$. This guarantees the convergence of the Fourier series in $(2.25)$ below. Because of $\left(T_{-1}^{0}, f\right)_{\mathcal{H}}=0$, condition $(2.11)_{2}$ is satisfied. Equations (2.23) hold and therefore we have, using $(2.4)_{3}$,

$$
\begin{gathered}
\left.\partial_{r} \Psi(r .)\right|_{S^{2}} \underset{r \searrow 1}{\stackrel{\mathcal{H}}{\longrightarrow}}-\sum_{n=1}^{\infty} \sum_{k=-n}^{n}(n+1) c_{n k} Y_{n}^{k}=f D_{r} . \\
\left.\sin \theta \partial_{\theta} \Psi(r .)\right|_{S^{2}} \underset{r \searrow 1}{\stackrel{\mathcal{H}}{\longrightarrow}} \sum_{n=0}^{\infty} \sum_{k=-n}^{n}\left(c_{n-1, k}(n--1) N_{n}^{k}-c_{n+1, k}(n+2) N_{n+1}^{k}\right) Y_{n}^{k} \\
\left.\partial_{\varphi} \Psi(r .)\right|_{S^{2}} \underset{r \searrow 1}{\stackrel{\mathcal{H}}{\longrightarrow}} \sum_{n=1}^{\infty} \sum_{k=-n}^{n} i k c_{n k} Y_{n}^{k} \sin \theta .
\end{gathered}
$$


Condition $\left(T_{n}^{k}, f\right)_{\mathcal{H}}=0$ in $(2.22)$ yields

$$
\begin{aligned}
\int_{S^{2}} f D_{\theta} \sin \theta Y_{n}^{k^{*}} d \Omega & =-\int_{S^{2}} f D_{r}\left(\frac{n-1}{n} N_{n}^{k} Y_{n-1}^{k^{*}}-N_{n+1}^{k} Y_{n+1}^{k^{*}}\right) d \Omega \\
& =\int_{S^{2}} \sum_{n^{\prime}, k^{\prime}}\left(n^{\prime}+1\right) c_{n^{\prime} k^{\prime}} Y_{n^{\prime}}^{k^{\prime}}\left(\frac{n-1}{n} N_{n}^{k} Y_{n-1}^{k^{*}}-N_{n+1}^{k} Y_{n+1}^{k}\right) d \Omega \\
& =c_{n-1, k}(n-1) N_{n}^{k}-c_{n+1, k}(n+2) N_{n+1}^{k},
\end{aligned}
$$

where we made use of $(2.25)_{1}$ in the second equation. From $(2.25)_{2}$ we now obtain

$$
\left.\sin \theta \partial_{\theta} \Psi(r .)\right|_{S^{2}} \underset{r \searrow 1}{\stackrel{\mathcal{H}}{\longrightarrow}} f D_{\theta} \sin \theta
$$

and by an analogous calculation, using condition $\left(S_{n}^{k}, f\right)_{\mathcal{H}}=0$ in $(2.22)$,

$$
\left.\partial_{\varphi} \Psi(r .)\right|_{S^{2}} \underset{r \searrow 1}{\stackrel{\mathcal{H}}{\longrightarrow}} f D_{\varphi} \sin \theta
$$

Axisymmetry simplifies the situation considerably. The axisymmetric Problem $\mathrm{P}_{3}^{a}$ follows from Problem $\mathrm{P}_{3}$ by the assumption $D_{r}=D_{r}(\theta), D_{\theta}=D_{\theta}(\theta)$ and $D_{\varphi}=0$. This implies $\partial_{\varphi} \Psi \equiv 0$. In Theorem 2.3 the conditions $\left(T_{n}^{k}, f\right)_{\mathcal{H}}=0$ reduce to those for $k=0$ and the conditions $\left(S_{n}^{k}, f\right)_{\mathcal{H}}=0$ can be omitted altogether. After a change of variables the appropriate Hilbert space is now $\mathcal{H}:=L^{2}((-1,1))$. It is, furthermore, convenient to replace the system $\left\{T_{n}^{0}: n \in \mathbb{N}_{0} \cup\{-1\}\right\}$ by an equivalent one $\left\{\mathcal{T}_{n}: n \in \mathbb{N}_{0}\right\}$. We then have:

Corollary 2.5. Let $\mathcal{D}_{r}, \mathcal{D}_{\theta} \in C^{0}([-1,+1], \mathbb{R}), g \in L^{2}((-1,1))$, and set

$$
D_{r}:=\mathcal{D}_{r}(\cos \theta), \quad D_{\theta}:=\mathcal{D}_{\theta}(\cos \theta) \sin \theta, \quad D_{\varphi}:=0, \quad f:=g(\cos \theta) .
$$

Moreover, define for $n \in \mathbb{N}$

$$
\begin{aligned}
& \mathcal{T}_{0}:=\mathcal{D}_{r} \\
& \mathcal{T}_{n}:=(n+1)\left(P_{n+1}-P_{n-1}\right) \mathcal{D}_{\theta}+(2 n+1) P_{n} \mathcal{D}_{r}
\end{aligned}
$$

where $\left(P_{n}\right)_{n}$ are the Legendre polynomials. Let

$$
W_{\mathbf{D}}:=\overline{\operatorname{Span}\left\{\mathcal{T}_{n}: n \in \mathbb{N}_{0}\right\}}
$$

Then $f$ is a solution of the axisymmetric Problem $\mathrm{P}_{3}^{a}$ if and only if $g \in W_{\mathbf{D}}^{\perp}$, i.e., $\forall n \in \mathbb{N}_{0}$ :

$$
\int_{-1}^{+1} g \mathcal{T}_{n} d x=0
$$


Proof. Since $\int_{S^{2}} u Y_{n}^{k^{*}} d \Omega=0$ for $k \neq 0$ and each $u \in L^{2}\left(S^{2}\right)$ independent of $\varphi$, all conditions in Eq. (2.22) of Theorem 2.4 with $k \neq 0$ can be omitted. We define for $n \in \mathbb{N}$

$$
\begin{aligned}
\tilde{T}_{0}(x) & :=\mathcal{D}_{r}(x), \\
\tilde{T}_{1}(x) & :=\left(1-x^{2}\right) \mathcal{D}_{\theta}(x)-x \mathcal{D}_{r}(x), \\
\tilde{T}_{n+1}(x) & :=(2 n+1)\left(1-x^{2}\right) \mathcal{D}_{\theta}(x) P_{n}(x) \\
& +\mathcal{D}_{r}(x)\left((n-1) P_{n-1}(x)-(n+1) P_{n+1}(x)\right)
\end{aligned}
$$

and obtain for $n \in \mathbb{N}_{0} \cup\{-1\}$

$$
T_{n}^{0}(\theta)=c(n) \tilde{T}_{n+1}(\cos \theta)
$$

where $c(n) \neq 0$ is independent of $x$ and $\theta$. With Eq. (A.4) it is easy to verify that for $n \in \mathbb{N}$ :

$$
\tilde{T}_{0}=\mathcal{T}_{0}, \quad \tilde{T}_{1}=\mathcal{T}_{1}, \quad \tilde{T}_{n+1}=(n-1) \mathcal{T}_{n-1}-(n+1) \mathcal{T}_{n+1}
$$

Thus, in this case, (2.22) is equivalent to (2.27).

3. Some results in the 2-dimensional case. This section deals with nowhere vanishing direction fields $\mathbf{D}$, which can conveniently be parametrized by a rotation function $\phi(\varphi)$ representing the polar angle of $\mathbf{D}$ in the coordinate system $\left\{\mathbf{e}_{r}(\varphi), \mathbf{e}_{\varphi}(\varphi)\right\}$. Theorem 3.1 determines the solution space of Problem $\mathrm{P}_{2}$ in the case of constant rotation rate $\phi^{\prime}(\varphi)$, which refers just to the $2^{N}$-pole fields $\mathbf{D}_{N}$. In the case of nonconstant rotation rate, a perturbation result is formulated in Theorem 3.3. Theorem 3.4, finally, provides uniqueness for the fields $\mathbf{D}_{N}$ if stronger decay conditions at infinity are required.

Nowhere vanishing direction fields $\mathbf{D} \in C^{1}\left(S^{1}, \mathbb{R}^{2}\right)$ can be normalized to one, $|\mathbf{D}|^{2}=$ $D_{r}^{2}+D_{\varphi}^{2}=1$, which allows the parametrization

$$
D_{r}(\varphi)=\cos \phi(\varphi), \quad D_{\varphi}(\varphi)=\sin \phi(\varphi)
$$

by a rotation function $\phi \in C^{1}(\mathbb{R}, \mathbb{R})$ with $\phi(\varphi+2 \pi)=\phi(\varphi)+2 \pi N, N \in \mathbb{Z}$. The condition (2.13) in Theorem 2.3 then takes the form

$$
\int_{-\pi}^{\pi} \cos (n \varphi+\phi(\varphi)) f(\varphi) d \varphi=\int_{-\pi}^{\pi} \sin (n \varphi+\phi(\varphi)) f(\varphi) d \varphi=0, \quad n \in \mathbb{N}_{0},
$$

or, in complex notation,

$$
\int_{-\pi}^{\pi} e^{i \phi(\varphi)} e^{i n \varphi} f(\varphi) d \varphi=\int_{-\pi}^{\pi} e^{-i \phi(\varphi)} e^{-i n \varphi} f(\varphi) d \varphi=0, \quad n \in \mathbb{N}_{0} .
$$

The following theorem specifies the solution space of Problem $\mathrm{P}_{2}$ in the case of constant rotation rate $\phi^{\prime}(\varphi)=N=$ const, $N \in \mathbb{Z} . N$ measures the number of turns of $\mathbf{D}$ in the coordinate system $\left\{\mathbf{e}_{r}(\varphi), \mathbf{e}_{\varphi}(\varphi)\right\}$ while $\varphi$ is running through the interval $(-\pi, \pi)$. It relates to the rotation number $r$, which is measured with respect to the fixed coordinate system $\left\{\mathbf{e}_{x}, \mathbf{e}_{y}\right\}$ by $r=N+1$. Note that the rotation function $\phi(\varphi)=\phi_{0}+N \varphi$ corresponds for $\phi_{0}=0$ and $\phi_{0}=\pi / 2$ to the multipole fields $\mathbf{D}_{N}=\cos (N \varphi) \mathbf{e}_{r}+$ $\sin (N \varphi) \mathbf{e}_{\varphi}$ and $\mathbf{D}_{N}=-\sin (N \varphi) \mathbf{e}_{r}+\cos (N \varphi) \mathbf{e}_{\varphi}$, respectively. 
Theorem 3.1. Let $\mathbf{D}(\varphi)=\cos \left(\phi_{0}+N \varphi\right) \mathbf{e}_{r}+\sin \left(\phi_{0}+N \varphi\right) \mathbf{e}_{\varphi}$ with $N \in \mathbb{Z}$ and $f \in$ $L^{2}\left(S^{1}\right)$. Then, $f$ solves Problem $\mathrm{P}_{2}$ if and only if $f \in V_{\mathbf{D}}^{\perp}:=\operatorname{Span}\left\{e^{i n \varphi}:-(N-1) \leq\right.$ $n \leq N-1\}$. In particular, $V_{\mathbf{D}}^{\perp}=\{0\}$ if $N \leq 0$ and $\operatorname{dim} V_{\mathbf{D}}^{\perp}=2(N-1)+1$ if $N>0$.

Proof. Let $f$ be expanded in the complete orthonormal system $\left\{\frac{e^{i \nu \varphi}}{\sqrt{2 \pi}}: \nu \in \mathbb{Z}\right\}$ in $L^{2}\left(S^{1}\right)$ :

$$
f=\sum_{\nu \in \mathbb{Z}} f_{\nu} \frac{e^{i \nu \varphi}}{\sqrt{2 \pi}} .
$$

Inserting (3.3) in (3.2), Theorem 2.3 furnishes for $n \in \mathbb{N}_{0}$

$$
\sum_{\nu \in \mathbb{Z}} f_{\nu} \int_{-\pi}^{\pi} e^{i(N+n+\nu) \varphi} d \varphi=\sum_{\nu \in \mathbb{Z}} f_{\nu} \int_{-\pi}^{\pi} e^{-i(N+n-\nu) \varphi} d \varphi=0,
$$

and thus

$$
f_{n+N}=f_{-n-N}=0, \quad n \in \mathbb{N}_{0}
$$

For the next theorem we need a result on the completeness of a perturbed complete orthonormal system that is due to Paley \& Wiener (1973):

LEMMA 3.2. Let $\left\{\psi_{n}: n \in \mathbb{Z}\right\}$ be a complete orthonormal system in $L^{2}((a, b))$ and let $\left\{\chi_{n}: n \in \mathbb{Z}\right\} \subset L^{2}((a, b))$. Let, furthermore, the inequality

$$
\left\|\sum_{n=-N}^{N} a_{n}\left(\psi_{n}-\chi_{n}\right)\right\|^{2} \leq \Theta \sum_{n=-N}^{N}\left|a_{n}\right|^{2}
$$

hold for arbitrary $N \in \mathbb{N}_{0}, a_{n} \in \mathbb{C},|n| \leq N$, and with $0<\Theta<1$ independent of $N$ and $a_{n}$. Then the system $\left\{\chi_{n}: n \in \mathbb{Z}\right\}$ is linearly independent and complete; i.e., for any $f \in L^{2}$ exist coefficients $b_{n}, n \in \mathbb{Z}$ such that

$$
f=\sum_{n=-\infty}^{\infty} b_{n} \chi_{n}
$$

There exists, moreover, another complete system $\left\{\tilde{\chi}_{n}: n \in \mathbb{Z}\right\} \subset L^{2}((a, b))$, which is dual to $\left\{\chi_{n}: n \in \mathbb{Z}\right\}$, i.e., $\left(\chi_{n}, \tilde{\chi}_{m}\right)_{\mathcal{H}}=\delta_{n m}$.

Proof. cf. Theorem XXXVII in (Paley \& Wiener 1973).

If the rotation rate is not constant the rotation function is decomposed according to

$$
\phi(\varphi)=\phi_{0}+N \varphi+\tilde{\phi}(\varphi) \quad \text { where } \quad \tilde{\phi}(\varphi+2 \pi)=\tilde{\phi}(\varphi), \quad \int_{-\pi}^{\pi} \tilde{\phi}(\varphi) d \varphi=0, \quad N \in \mathbb{Z} .
$$

$\tilde{\phi}^{\prime}(\varphi)$ is then the deviation of the rotation rate from its mean value. For small deviations we have the following result:

TheOREM 3.3. Let $\phi \in C^{2}(\mathbb{R}, \mathbb{R})$ be the rotation function of a direction field $\mathbf{D}$ according to (3.1) with decomposition (3.5). Let $N \in \mathbb{Z}$ be the mean rotation rate and $\tilde{\phi}$ satisfying the conditions

$$
\left\|\tilde{\phi}^{\prime}\right\|<\epsilon, \quad\left\|\tilde{\phi}^{\prime \prime}\right\|<1
$$


with $\epsilon>0$ such that $\left(\frac{5}{3} \pi+\frac{1}{2 \pi}\right) \epsilon^{2}+\frac{2}{3} \pi \epsilon<1$ and $\|\cdot\|$ denoting the norm in $\mathcal{H}:=$ $L^{2}((-\pi, \pi))$. Then, there holds for the solution space $V_{\mathbf{D}}^{\perp}$ of Problem $\mathrm{P}_{2}$ :

$$
\operatorname{dim} V_{\mathbf{D}}^{\perp}=\left\{\begin{array}{cl}
0 & \text { if } \quad N \leq 0, \\
2(N-1)+1 & \text { if } \quad N>0 ;
\end{array}\right.
$$

i.e., the result of Theorem 3.1 is stable under small perturbations of the rotation rate.

Proof. We start with some auxiliary estimates.

(i) With $\int_{-\pi}^{\pi} e^{i(m-n) x} d x=2 \pi \delta_{m n}, m, n \in \mathbb{Z}$ and the Fourier series

$$
2 \sum_{\nu \in \mathbb{Z} \backslash\{0\}} \frac{\cos \nu x}{\nu^{2}}=\sum_{\nu \in \mathbb{Z} \backslash\{0\}} \frac{e^{i \nu x}}{\nu^{2}}=\frac{x^{2}}{2}-\pi x+\frac{\pi^{2}}{3}
$$

on $(0,2 \pi)$, one obtains for $a_{n} \in \mathbb{C},|n| \leq N$ :

$$
\begin{aligned}
& \sum_{\substack{m, n=-N \\
m \neq n}}^{N} \frac{\left|a_{m}\right|\left|a_{n}\right|}{(m-n)^{2}}=\sum_{\substack{m, n=-N \\
m \neq n}}^{N}\left|a_{m}\right|\left|a_{n}\right| \sum_{\nu=-\infty}^{\infty} \frac{1}{\nu^{2}} \cdot \frac{1}{2 \pi} \int_{-\pi}^{\pi} e^{i(m-n-\nu) x} d x \\
= & \frac{1}{2 \pi} \int_{-\pi}^{\pi} \sum_{m, n=-N}^{N}\left|a_{m}\right|\left|a_{n}\right| e^{i(m-n) x} \sum_{\substack{\nu=-\infty \\
\nu \neq 0}}^{\infty} \frac{e^{-i \nu x}}{\nu^{2}} d x \\
\leq & \frac{1}{2 \pi} \int_{-\pi}^{\pi}\left|\sum_{n=-N}^{N}\right| a_{n}\left|e^{i n x}\right|^{2} \cdot \max _{[0, \pi]}\left(\frac{1}{2} x^{2}-\pi x+\frac{\pi^{2}}{3}\right) d x \\
= & \frac{\pi^{2}}{3} \sum_{m, n=-N}^{N}\left|a_{m}\right|\left|a_{n}\right| \frac{1}{2 \pi} \int_{-\pi}^{\pi} e^{i(m-n) x} d x=\frac{\pi^{2}}{3} \sum_{n=-N}^{N}\left|a_{n}\right|^{2} .
\end{aligned}
$$

(ii) Since $\tilde{\phi} \in C^{1}(\mathbb{R}, \mathbb{C})$ satisfies the conditions (3.5), the following Poincaré-type inequality holds:

$$
\int_{-\pi}^{\pi}|\tilde{\phi}(x)|^{2} d x \leq \int_{-\pi}^{\pi}\left|\tilde{\phi}^{\prime}(x)\right|^{2} d x
$$

Let us define $\lambda(x):=4 \sin ^{2}(\tilde{\phi}(x) / 2)$ and $\varrho_{ \pm}(x):=e^{ \pm i \tilde{\phi}(x)} \lambda(x)$. We then have with (3.6) and (3.9):

$$
\begin{gathered}
\int_{-\pi}^{\pi} \lambda(x) d x \leq\|\tilde{\phi}\|^{2} \leq\left\|\tilde{\phi}^{\prime}\right\|^{2} \leq \epsilon^{2}, \\
\int_{-\pi}^{\pi}\left|\lambda(x)^{\prime \prime}\right| d x \leq 2\left(\left\|\tilde{\phi}^{\prime \prime}\right\|\|\tilde{\phi}\|+\left\|\tilde{\phi}^{\prime}\right\|^{2}\right) \leq 2\left(\left\|\tilde{\phi}^{\prime \prime}\right\|\left\|\tilde{\phi}^{\prime}\right\|+\left\|\tilde{\phi}^{\prime}\right\|^{2}\right) \leq 2 \epsilon(1+\epsilon), \\
\int_{-\pi}^{\pi}\left|\varrho_{ \pm}(x)\right| d x \leq \int_{-\pi}^{\pi}\left|\lambda(x)^{\prime \prime}\right| d x+4 \int_{-\pi}^{\pi}\left|\left(\tilde{\phi}^{\prime}\right)^{2} \sin \tilde{\phi}\right| d x \\
\leq 2 \epsilon(1+\epsilon)+4 \epsilon^{2}+4\left\|\tilde{\phi}^{\prime \prime}\right\|\left\|\sin ^{2}(\tilde{\phi} / 2)\right\|+4 \epsilon^{2} \\
\leq 2 \epsilon(1+\epsilon)+4 \epsilon^{2}+2\|\tilde{\phi} \sin (\tilde{\phi} / 2)\|+4 \epsilon^{2} \leq 10 \epsilon^{2}+4 \epsilon .
\end{gathered}
$$

(iii) The next step is to prove the system $\left\{e^{i c_{n}} e^{i n \varphi} e^{i \sigma(n) \tilde{\phi}(\varphi)}: n \in \mathbb{Z}\right\}$ with $c_{n}:=$ $\sigma(n) \phi_{0}$ and $\sigma(n)=1$ if $n \geq 0$ and otherwise $\sigma(n)=-1$ to be complete in $L^{2}((-\pi, \pi))$. 
For this purpose we set $a:=-\pi, b:=\pi, \psi_{n}(x):=\frac{1}{\sqrt{2 \pi}} e^{i c_{n}} e^{i n x}$ and $\chi_{n}(x):=$ $\psi_{n}(x) e^{i \sigma(n) \tilde{\phi}(x)}$ in Lemma 3.2. We then have to prove that

$$
\frac{1}{2 \pi} \int_{-\pi}^{\pi}\left|\sum_{n=-N}^{N} \tilde{a}_{n}\left(e^{i \sigma(n) \tilde{\phi}(x)}-1\right) e^{i n x}\right|^{2} d x \leq \Theta \sum_{n=-N}^{N}\left|\tilde{a}_{n}\right|^{2}
$$

where $N \in \mathbb{N}_{0}$ and $\tilde{a}_{n}=a_{n} e^{i c_{n}} \in \mathbb{C},|n| \leq N$ are arbitrary, and $0<\Theta<1$ is independent of $N$ and $\tilde{a}_{n}$. Let us define

$$
F_{m n}(x):=\left(e^{-i \sigma(m) \tilde{\phi}(x)}-1\right)\left(e^{i \sigma(n) \tilde{\phi}(x)}-1\right) .
$$

For $\sigma(m)=\sigma(n)$ follows $F_{m n}(x)=\lambda(x)$; otherwise $F_{m n}(x)=-\varrho_{ \pm}(x)$. Thus $(3.10)$ implies the estimate

$$
\int_{-\pi}^{\pi}\left|F_{m n}^{\prime \prime}(x)\right| d x \leq 10 \epsilon^{2}+4 \epsilon .
$$

Expanding the left-hand side in (3.11) and using (3.8), (3.10) ${ }_{1}$, and (3.12) furnishes:

$$
\begin{aligned}
& \frac{1}{2 \pi} \int_{-\pi}^{\pi}\left\{\sum_{\substack{m, n=-N \\
m \neq n}}^{N} a_{m}^{*} a_{n} F_{m n}(x) e^{i(n-m) x}+\sum_{n=-N}^{N}\left|a_{n}\right|^{2} \lambda(x)\right\} d x \\
& =\frac{1}{2 \pi} \int_{-\pi}^{\pi}\left\{-\sum_{\substack{m, n=-N \\
m \neq n}}^{N} a_{m}^{*} a_{n} F_{m n}^{\prime \prime}(x) \frac{e^{i(n-m) x}}{(m-n)^{2}}+\sum_{n=-N}^{N}\left|a_{n}\right|^{2} \lambda(x)\right\} d x \\
& \leq \frac{1}{2 \pi}\left\{\int_{-\pi}^{\pi}\left|F_{m n}^{\prime \prime}(x)\right| d x \sum_{\substack{m, n=-N \\
m \neq n}}^{N} \frac{\left|a_{m}\right|\left|a_{n}\right|}{(m-n)^{2}}+\int_{-\pi}^{\pi} \lambda(x) d x \sum_{n=-N}^{N}\left|a_{n}\right|^{2}\right\} \\
& \leq \frac{1}{2 \pi}\left(\frac{\pi^{2}}{3}\left(10 \epsilon^{2}+4 \epsilon\right)+\epsilon^{2}\right) \sum_{\substack{n=-N \\
m=-n}}^{N}\left|a_{n}\right|^{2} .
\end{aligned}
$$

This proves (3.11).

(iv) We now prove (3.7). According to Theorem 2.2 and Eqs. (3.2), $V_{\mathbf{D}}^{\perp}$ is given by $V_{\mathbf{D}}^{\perp}=\overline{\operatorname{Span}\left\{\tilde{T}_{n}, \tilde{S}_{n}: n \in \mathbb{N}_{0}\right\}}{ }^{\perp}$ with

$$
\begin{aligned}
& \tilde{T}_{n}:=e^{i \phi_{0}} e^{i(n+N) \varphi} e^{i \tilde{\phi}(\varphi)}, \\
& \tilde{S}_{n}:=e^{-i \phi_{0}} e^{-i(n+N) \varphi} e^{-i \tilde{\phi}(\varphi)} .
\end{aligned}
$$

In the case $N \leq 0$ there is obviously

$$
\overline{\operatorname{Span}\left\{e^{i \sigma(n) \phi_{0}} e^{i n \varphi} e^{i \sigma(n) \tilde{\phi}(\varphi)}: n \in \mathbb{Z}\right\}} \subset \overline{\operatorname{Span}\left\{\tilde{T}_{n}, \tilde{S}_{n}: n \in \mathbb{N}_{0}\right\}}
$$

and from (iii) immediately follows $V_{\mathbf{D}}^{\perp}=\{0\}$ and hence $(3.7)_{1}$. If $N>0$, let $\chi_{n}(x)$ be defined as in (iii) and let $\tilde{\chi}_{n}(x)$ be the complete dual system according to Lemma 3.2. Obviously, there is $\tilde{T}_{n}=\chi_{n+N}$ and $\tilde{S}_{n}=\chi_{-n-N}$ and the system $\left\{\chi_{n}: n \in \mathbb{N}_{0}\right\}=\left\{\chi_{n}\right.$ : $|n| \leq N-1\} \cup\left\{\tilde{T}_{n}, \tilde{S}_{n}: n \in \mathbb{N}_{0}\right\}$ is then complete according to (iii). We have, therefore,

$$
V_{\mathbf{D}}^{\perp}=\overline{\operatorname{Span}\left\{\tilde{T}_{n}, \tilde{S}_{n}: n \in \mathbb{N}_{0}\right\}}{ }^{\perp}=\operatorname{Span}\left\{\tilde{\chi}_{n}:|n| \leq N-1\right\}
$$

with $\operatorname{dim} V_{\mathbf{D}}^{\perp}=2(N-1)+1$. This proves $(3.7)_{2}$. 
In the case of constant rotation rate, it follows from Theorem 3.1 that all solutions $f$ (and hence $\Psi$ ) have finite expansions in the system $\left\{e^{i \nu \varphi}: \nu \in \mathbb{Z}\right\}$. So, allowing only functions with finite expansions as admissible solutions of Problem $\mathrm{P}_{2}$ does not imply uniqueness. Other restrictions, however, can enforce uniqueness:

Theorem 3.4. Let $\mathbf{D}(\varphi)=\cos \left(\phi_{0}+N \varphi\right) \mathbf{e}_{r}+\sin \left(\phi_{0}+N \varphi\right) \mathbf{e}_{\varphi}$ with $N \in \mathbb{N}$. Then the modified Problem $\mathrm{P}_{2}$ where $\Psi$ has to additionally satisfy

$$
|\Psi|=O\left(|\mathbf{x}|^{-N}\right) \quad \text { for }|\mathbf{x}| \rightarrow \infty
$$

is uniquely solvable.

Proof. According to Theorem 3.1, a solution $f$ of $\mathrm{P}_{2}$ allows the representation

$$
f(\varphi)=\sum_{\nu=-(N-1)}^{N-1} f_{\nu} e^{i \nu \varphi}
$$

with $f_{\nu} \in \mathbb{C}$. The corresponding potential is given in (2.14) with coefficients $a_{n}, b_{n}$ determined by Eqs. (2.16) and (2.17). Condition (3.13) implies $a_{n}=b_{n}=0$ for $n=$ $1, \ldots, N-1$. Thus, Eqs. (2.16), (2.17) imply for $n=1, \ldots, N-1$ :

$$
\begin{aligned}
& \int_{-\pi}^{\pi} \sum_{\nu=-(N-1)}^{N-1} f_{\nu} e^{i \nu \varphi} \cos \left(\phi_{0}+N \varphi\right) \cos n \varphi d \varphi=0, \\
& \int_{-\pi}^{\pi} \sum_{\nu=-(N-1)}^{N-1} f_{\nu} e^{i \nu \varphi} \sin \left(\phi_{0}+N \varphi\right) \sin n \varphi d \varphi=0, \\
& \int_{-\pi}^{\pi} \sum_{\nu=-(N-1)}^{N-1} f_{\nu} e^{i \nu \varphi} \cos \left(\phi_{0}+N \varphi\right) \sin n \varphi d \varphi=0, \\
& \int_{-\pi}^{\pi} \sum_{\nu=-(N-1)}^{N-1} f_{\nu} e^{i \nu \varphi} \sin \left(\phi_{0}+N \varphi\right) \cos n \varphi d \varphi=0 .
\end{aligned}
$$

By linear combination we arrive at

$$
\sum_{\nu=-(N-1)}^{N-1} f_{\nu} \int_{-\pi}^{\pi} e^{i(\nu \pm N \pm n) \varphi} d \varphi=0, \quad n=1, \ldots, N-1
$$

(one equation for every sign combination), and by this

$$
f_{N-n}=f_{-N+n}=0, \quad n=1, \ldots, N-1 .
$$

The only solution is thus $f=$ const.

4. Some results in the 3-dimensional axisymmetric case. This section deals with axisymmetric 3-dimensional direction fields $\mathbf{D}$, in particular, with those arising from axisymmetric $2^{N}$-pole fields $\mathbf{D}_{N}$. Upper bounds on the dimension of the solution space $W_{\mathbf{D}}$ for arbitrary polynomial direction fields can easily be derived. It is, however, much harder to determine the precise dimension. This is done for the direction fields $\mathbf{D}_{N}$. The cases $N=1$ and 2 are treated explicitly and the general case is dealt with in Theorem 4.3. The Lemmata 4.1, 4.2, and 4.4 present auxiliary results. Theorem 4.5 
extends the result of Theorem 4.3 for $N=1$ to all fields which are close in a certain norm to $\mathbf{D}_{1}$. Theorem 4.7 and Remark 4.8 present possibilities to enforce uniqueness for direction fields $\mathbf{D}_{N}$ with $N>1$. Finally, we investigate exemplarily and on a numerical basis the dependence of $\operatorname{dim} W_{\mathbf{D}}^{\perp}$ on the rotation number of $\mathbf{D}$ and formulate a conjecture in this connection.

Let us consider the situation of Corollary 2.5. If $\mathcal{D}_{r}, \mathcal{D}_{\theta}$ are polynomials, then the $\mathcal{T}_{n}$ are also polynomials. Each $\mathcal{T}_{n}$ is then a finite linear combination of the $\left(P_{n}\right)_{n \in \mathbb{N}_{0}}$. Moreover, if each $\mathcal{T}_{n}$ has degree $n+N, N \in \mathbb{N}$, one can derive a recurrence relation for the Fourier coefficients $\sigma_{n} \in \mathbb{C}, n \in \mathbb{N}_{0}$ of $g$, where

$$
g=\sum_{n=0}^{\infty} \sigma_{n} \tilde{P}_{n}
$$

and where $\tilde{P}_{n}$ are the normalized Legendre-Polynomials (see Appendix A). Writing the $\mathcal{T}_{n}$ in terms of $\tilde{P}_{n}$ in the form

$$
\begin{aligned}
\mathcal{T}_{n} & =\sqrt{2(2 n+1)}\left(\left(\alpha_{n} \tilde{P}_{n+1}-\beta_{n} \tilde{P}_{n-1}\right) \mathcal{D}_{\theta}+\tilde{P}_{n} \mathcal{D}_{r}\right), \\
\alpha_{n} & :=\frac{n+1}{\sqrt{(2 n+1)(2 n+3)}}, \quad \beta_{n}:=\frac{n+1}{\sqrt{(2 n-1)(2 n+1)}},
\end{aligned}
$$

and repeatedly using (A.7), one can easily compute coefficients $\nu_{n k}, n \in \mathbb{N}_{0}, k=$ $-N, \ldots, N$ such that

$$
\mathcal{T}_{n}=\sum_{k=-N}^{N} \nu_{n k} \tilde{P}_{n+k} \quad \text { with } \nu_{n k}=0 \text { if } k<-n \text { and } \nu_{n N} \neq 0, \quad n \in \mathbb{N}_{0} .
$$

Note that because of $\lim _{n \rightarrow \infty} \alpha_{n}=\lim _{n \rightarrow \infty} \beta_{n}=\frac{1}{2}$, there exist $\nu_{k} \in \mathbb{R}$ such that $\lim _{n \rightarrow \infty} \nu_{n k}=\nu_{k}, k=-N, \ldots, N$.

Now, condition (2.27) yields $\forall n \in \mathbb{N}_{0}$ :

$$
\sigma_{n+N}=-\frac{1}{\nu_{n N}} \sum_{k=-N}^{N-1} \nu_{n k} \sigma_{n+k}
$$

i.e., only the initial values $\sigma_{0}, \ldots, \sigma_{N-1}$ are not determined. Therefore, we can immediately conclude $\operatorname{dim} W_{D}^{\perp} \leq N$. In general, it is not clear that for each selection of $\sigma_{0}, \ldots, \sigma_{N-1}$, the sequence $\left(\sigma_{n}\right)_{n \in \mathbb{N}_{0}}$ defined by (4.3) yields a convergent Fourier series for $g$. To investigate this, the following lemma is a first criterion.

Lemma 4.1. Consider the recurrence relation (as in (4.3))

$$
\sum_{k=-N}^{+N} \nu_{n k} \sigma_{n+k}=0, \quad n \in \mathbb{N}_{0}
$$

with $\nu_{n k}=0$ if $k<-n, \nu_{n N} \neq 0, \nu_{n k} \rightarrow \nu_{k}$ for $n \rightarrow \infty, \nu_{N} \neq 0$, and the related polynomial

$$
Q(X):=\sum_{k=-N}^{+N} \nu_{k} X^{k+N} \in \mathbb{R}[X]
$$


of degree $2 N$. If $|z|<1$ for all complex zeroes $z$ of $Q(X)$, then the series $\sum_{n=0}^{\infty}\left|\sigma_{n}\right|^{2}$ and therefore the Fourier series $g=\sum_{n=0}^{\infty} \sigma_{n} \tilde{P}_{n}$ converges for arbitrary initial values $\sigma_{0}, \ldots, \sigma_{N-1} \in \mathbb{C}$.

Proof. (i) Relation (4.4) can be written in matrix form

$$
\left(\begin{array}{c}
\sigma_{n-N+1} \\
\sigma_{n-N+2} \\
\vdots \\
\sigma_{n+N-1} \\
\sigma_{n+N}
\end{array}\right)=\left(\begin{array}{cccccc}
0 & 1 & 0 & \ldots & 0 & 0 \\
0 & 0 & 1 & \ldots & 0 & 0 \\
\vdots & \vdots & \vdots & & \vdots & \vdots \\
0 & 0 & 0 & \ldots & 0 & 1 \\
\frac{\nu_{n,-N}}{-\nu_{n N}} & \frac{\nu_{n,-N+1}}{-\nu_{n N}} & \frac{\nu_{n,-N+2}}{-\nu_{n N}} & \ldots & \frac{\nu_{n, N-2}}{-\nu_{n}} & \frac{\nu_{n, N-1}}{-\nu_{n N}}
\end{array}\right)\left(\begin{array}{c}
\sigma_{n-N} \\
\sigma_{n-N+1} \\
\vdots \\
\sigma_{n+N-2} \\
\sigma_{n+N-1}
\end{array}\right)
$$

where the $2 N \times 2 N$-matrix in (4.6) shall be denoted by $S_{n} \in \mathbb{R}^{2 N \times 2 N}$. By iteration of (4.6) we obtain

$$
\left(\begin{array}{c}
\sigma_{n+N} \\
\sigma_{n+N+1} \\
\vdots \\
\sigma_{n+3 N-2} \\
\sigma_{n+3 N-1}
\end{array}\right)=S_{n+2 N-1} \cdot \ldots \cdot S_{n+1} \cdot S_{n}\left(\begin{array}{c}
\sigma_{n-N} \\
\sigma_{n-N+1} \\
\vdots \\
\sigma_{n+N-2} \\
\sigma_{n+N-1}
\end{array}\right)
$$

and, therefore, convergence of $\sum_{n=0}^{\infty}\left|\sigma_{n}\right|^{2}$ is guaranteed if there are some $q \in(0,1)$ and some $m \in \mathbb{N}$ satisfying

$$
\forall n \in \mathbb{N}, n \geq n_{0}: \quad \operatorname{lub}_{2}\left(S_{n+2 m N-1} \cdot \ldots \cdot S_{n+2 N-1} \cdot \ldots \cdot S_{n+1} \cdot S_{n}\right) \leq q
$$

for sufficiently large $n_{0} \in \mathbb{N}$, where $l_{1} b_{2}$ is the matrix norm corresponding to the euclidean vector norm. Since the matrix norm depends continuously on the coefficients of the matrix, condition (4.7) is fulfilled if

$$
\operatorname{lub}_{2}\left(S^{2 m N}\right)<q, \quad \text { where } S:=\lim _{n \rightarrow \infty} S_{n} .
$$

(ii) Now assume $|z|<1$ for all zeroes $z$ of $Q(X)$. Since $Q(X)$ is the characteristic polynomial of $S$, we can choose $\mu \in(0,1)$ and $\epsilon>0$ such that

$$
\varrho(S):=\max \{|\lambda| \mid \lambda \text { is eigenvalue of } S\} \leq \mu-\epsilon .
$$

From spectral approximation theory (see, e.g., (Stoer \& Bulirsch 1978, Theorem (6.9.2))) it is well known that for each matrix $M \in \mathbb{R}^{2 N \times 2 N}$ and each $\epsilon>0$ there is some vector norm $|\cdot|_{\epsilon}$ in $\mathbb{R}^{2 N}$ satisfying

$$
\operatorname{lub}_{||_{\epsilon}}(M):=\sup _{|x|_{\epsilon}=1}|M x|_{\epsilon} \leq \varrho(M)+\epsilon .
$$

Since all matrix norms on $\mathbb{R}^{2 N \times 2 N}$ are equivalent, there is some $C_{\epsilon}>0$ with the property

$$
\forall M \in \mathbb{R}^{2 N \times 2 N}: \quad \operatorname{lub}_{2}(M) \leq C_{\epsilon} \operatorname{lub}_{|\cdot|_{\epsilon}}(M) .
$$

Using (4.9) and applying (4.10) and (4.11) on $M=S^{2 m N}$, we finally arrive at

$$
\operatorname{lub}_{2}\left(S^{2 m N}\right) \leq C_{\epsilon} \operatorname{lub}_{|\cdot|_{\epsilon}}\left(S^{2 m N}\right) \leq C_{\epsilon} \mu^{2 m N} \leq q
$$


for sufficiently large $m \in \mathbb{N}$. Thus, condition (4.8) is satisfied and part (i) of the proof yields the statement of the lemma.

The following lemma of Eneström and Kakeya is a useful tool in order to apply Lemma 4.1 .

Lemma 4.2. Let $N \in \mathbb{N}, \kappa_{0}, \ldots, \kappa_{N} \in \mathbb{R}_{+}$and $R(Z):=\sum_{k=0}^{N} \kappa_{k} Z^{k} \in \mathbb{R}[Z]$.

(a) If $\kappa_{N} \geq \kappa_{N-1} \geq \ldots \geq \kappa_{1} \geq \kappa_{0}>0$, then $|z| \leq 1$ for all complex zeroes $z$ of $R(Z)$.

(b) If $\kappa_{N}>\kappa_{N-1}>\ldots>\kappa_{1}>\kappa_{0}>0$, then $|z|<1$ for all complex zeroes $z$ of $R(Z)$.

Proof. For part (a) we refer to Kakeya (1912). (b) is a trivial consequence of (a).

4.1. Axisymmetric multipole fields. In this subsection we will show that for direction fields corresponding to $2^{N}$-pole fields, i.e.,

$$
\mathcal{D}_{r}^{N}(x)=(N+1) P_{N}(x), \quad \mathcal{D}_{\theta}^{N}(x)=P_{N}^{\prime}(x),
$$

the dimension of the solution space of Problem $\mathrm{P}_{3}^{a}, \operatorname{dim} W_{\mathbf{D}_{N}}^{\perp}$, equals $N$.

ExAmples. (a) The dipole field: Here we have

$$
\mathcal{D}_{r}^{1}(x)=2 x, \quad \mathcal{D}_{\theta}^{1}(x)=1,
$$

and therefore, using (A.7), (4.2),

$$
\mathcal{T}_{0}=2 P_{1}, \quad \mathcal{T}_{n}=\sqrt{2(2 n+1)}\left(3 \alpha_{n} \tilde{P}_{n+1}+\left(2 \alpha_{n-1}-\beta_{n}\right) \tilde{P}_{n-1}\right), \quad n \in \mathbb{N} .
$$

From condition (2.27) we obtain

$$
\sigma_{1}=0, \quad \sigma_{n+1}=-\frac{2 \alpha_{n-1}-\beta_{n}}{3 \alpha_{n}} \sigma_{n-1}, n \in \mathbb{N} .
$$

Since $2 \alpha_{n-1}-\beta_{n}=0$ iff $n=1$, we conclude $\sigma_{2 k}=0$ for every $k \in \mathbb{N}$ and since $\sigma_{1}=0$ we also have $\sigma_{2 k+1}=0$ for every $k \in \mathbb{N}_{0}$. Thus, we have the unique solution $g=\sigma_{0} \tilde{P}_{0}$.

(b) The quadrupole field: Let

$$
\mathcal{D}_{r}^{2}(x)=\frac{3}{2}\left(3 x^{2}-1\right), \quad \mathcal{D}_{\theta}^{2}(x)=3 x .
$$

In the same way as above we obtain

$$
\begin{aligned}
\mathcal{T}_{0} & =3 P_{2} \\
\mathcal{T}_{n} & =3 \sqrt{n+\frac{1}{2}} \\
& \times\left(5 \alpha_{n} \alpha_{n+1} \tilde{P}_{n+2}+\left(5 \alpha_{n}^{2}+3 \alpha_{n-1}^{2}-2 \alpha_{n-1} \beta_{n}-1\right) \tilde{P}_{n}+\left(3 \alpha_{n-1} \alpha_{n-2}-2 \alpha_{n-2} \beta_{n}\right) \tilde{P}_{n-2}\right),
\end{aligned}
$$

and, therefore,

$$
\begin{aligned}
\sigma_{2} & =0 \\
\sigma_{n+2} & =-\frac{5 \alpha_{n}^{2}+3 \alpha_{n-1}^{2}-2 \alpha_{n-1} \beta_{n}-1}{5 \alpha_{n} \alpha_{n+1}} \sigma_{n}-\frac{3 \alpha_{n-1} \alpha_{n-2}-2 \beta_{n} \alpha_{n-2}}{5 \alpha_{n} \alpha_{n+1}} \sigma_{n-2},
\end{aligned}
$$

where the coefficient in front of $\sigma_{n}$ vanishes iff $n=1$ and the coefficient in front of $\sigma_{n-2}$ vanishes iff $n=1$ or $n=2$. Thus, we have $\sigma_{2 k}=0$ for $k \in \mathbb{N}$ and

$$
g=\frac{\sigma_{0}}{\sqrt{2}}+\sum_{k=0}^{\infty} \sigma_{2 k+1} \tilde{P}_{2 k+1}
$$


where the $\sigma_{2 k+1}$ are uniquely determined by the choice of $\sigma_{1}$. With $\lim _{n \rightarrow \infty} \alpha_{n}=$ $\lim _{n \rightarrow \infty} \beta_{n}=1 / 2$ follows for the coefficients in (4.12):

$$
\begin{aligned}
\nu_{2} & =1, \\
\nu_{0} & =\lim _{n \rightarrow \infty}\left|\frac{5 \alpha_{n}^{2}+3 \alpha_{n-1}^{2}-2 \alpha_{n-1} \beta_{n}-1}{5 \alpha_{n} \alpha_{n+1}}\right|=\frac{2}{5}, \\
\nu_{-2} & =\lim _{n \rightarrow \infty}\left|\frac{3 \alpha_{n-1} \alpha_{n-2}-2 \beta_{n} \alpha_{n-2}}{5 \alpha_{n} \alpha_{n+1}}\right|=\frac{1}{5},
\end{aligned}
$$

i.e., the Lemmata 4.1 and 4.2 imply the convergence of the Fourier series for $g$. Thus, we have $\operatorname{dim} W_{\mathbf{D}_{2}}^{\perp}=2$. Note that in the case $\sigma_{1} \neq 0$, the corresponding solution $\Psi$ of the boundary value problem (2.11) has a nonvanishing dipole moment: Assume as in (2.5)

$$
\Psi=-\frac{1}{\sqrt{2 \pi}} \sum_{n=1}^{\infty} \frac{b_{n}}{n+1} r^{-(n+1)} \tilde{P}_{n}(\cos \theta), \quad \frac{\partial \Psi}{\partial r}=\frac{1}{\sqrt{2 \pi}} \sum_{n=1}^{\infty} b_{n} r^{-(n+2)} \tilde{P}_{n}(\cos \theta)
$$

From condition $(2.12)_{1}$ follows:

$$
b_{n}=\sqrt{2 \pi} \int_{-1}^{+1} g(x) \mathcal{D}_{r}(x) \tilde{P}_{n}(x) d x=\sqrt{2 \pi} \int_{-1}^{+1} \sum_{k=0}^{\infty} \sigma_{k} \tilde{P}_{k}(x) \tilde{P}_{n}(x) \mathcal{D}_{r}(x) d x .
$$

For the dipole moment $b_{1}$ we then obtain

$$
\begin{aligned}
b_{1} & =\sqrt{2 \pi} \int_{-1}^{+1} \sum_{k=0}^{\infty} \sigma_{k} \tilde{P}_{k}(x)\left(\frac{3}{2}\right)^{3 / 2} x\left(3 x^{2}-1\right) d x \\
& =\sqrt{2 \pi} \int_{-1}^{+1} \sum_{k=0}^{\infty} \sigma_{k} \tilde{P}_{k}(x)\left(\frac{9}{5} \sqrt{\frac{3}{7}} \tilde{P}_{3}(x)+\frac{6}{5} \tilde{P}_{1}(x)\right) d x \\
& =\sqrt{2 \pi}\left(\frac{9}{5} \sqrt{\frac{3}{7}} \sigma_{3}+\frac{6}{5} \sigma_{1}\right)=\sqrt{2 \pi} \frac{6}{5} \sigma_{1} .
\end{aligned}
$$

In the last equation we made use of (4.12) with the result $\sigma_{3}=0$.

The general case $N \in \mathbb{N}$ is treated in the following theorem:

THEOREM 4.3. Let $N \in \mathbb{N}$ and

$$
\mathcal{D}_{r}^{N}(x)=(N+1) P_{N}(x), \quad \mathcal{D}_{\theta}^{N}(x)=P_{N}^{\prime}(x) .
$$

Then $\operatorname{dim} W_{\mathbf{D}_{N}}^{\perp}=N$.

Proof. (i) Let $g=\sum_{k=0}^{\infty} \sigma_{k} \tilde{P}_{k}$. There are coefficients $\nu_{n k} \in \mathbb{R}$ with $n \in \mathbb{N}_{0}, k=$ $-N, \ldots, N$, such that

$$
\mathcal{T}_{n}(x)=\sum_{k=-N}^{N} \nu_{n k} \tilde{P}_{n+k}(x)
$$

where $\nu_{n k}=0$ if $k<-n$ and $\nu_{n N} \neq 0$. Let $\nu_{k}:=\lim _{n \rightarrow \infty} \nu_{n k}$, and note that $\nu_{N} \neq 0$. The condition $\left(\mathcal{T}_{n}, g\right)_{\mathcal{H}}=0$ (cf. (2.27)) then furnishes (4.4) with the corresponding polynomial $Q(X)$ as in (4.5). Obviously, we can conclude $\operatorname{dim} W_{\mathbf{D}_{N}}^{\perp} \leq N$. 
(ii) It turns out to be more convenient to use instead of (4.4) an alternative recurrence relation for the $\sigma_{n}$. From Eq. (4.4) follows immediately

$$
\sum_{k=-N}^{N} \nu_{n+2, k} \sigma_{n+2+k}-\frac{n}{n+2} \sum_{k=-N}^{N} \nu_{n k} \sigma_{n+k}=0
$$

which is equivalent to the condition

$$
\left(\tilde{T}_{n+2}, g\right)_{\mathcal{H}}=0, \text { where } \frac{1}{n+2} \tilde{T}_{n+2}=\frac{n}{n+2} \mathcal{T}_{n}-\mathcal{T}_{n+2}, \quad n \in \mathbb{N}_{0}
$$

(cf. (2.28)). Since $\lim _{n \rightarrow \infty} \nu_{n+2, k}=\lim _{n \rightarrow \infty} \nu_{n k}=\nu_{k}$ and $\lim _{n \rightarrow \infty} \frac{n}{n+2}=1$, the polynomial $\tilde{Q}(X) \in \mathbb{R}[X]$ related to (4.17) according to Lemma 4.1 is given by

$$
\tilde{Q}(X)=\left(X^{2}-1\right) Q(X) .
$$

(iii) Next, we want to determine explicitly the coefficients of the recurrence relation and its asymptotic values. The basic tools are relations of the type

$$
P_{N}(x) P_{n}(x)=\sum_{k}(2 k+1)\langle N n k\rangle P_{k}(x), \quad N, n, k \in \mathbb{N}_{0},
$$

expressing products of Legendre polynomials by finite sums of Legendre polynomials. The coefficients $\langle N n k\rangle$ are related to the Clebsch-Gordan coefficients resp. Wigner symbols appearing in the representation theory of the rotation group (cf. Appendix B). We note here only that $\langle N n k\rangle \geq 0$, the total symmetry of $\langle N n k\rangle$, and the property

$$
(N+n+k \in 2 \mathbb{Z} \wedge|N-n| \leq k \leq N+n) \Leftrightarrow\langle N n k\rangle \neq 0,
$$

which ensures the finiteness of the sum (4.20).

From (4.18), (2.26), (4.15), (A.5), (4.20), and (A.3) follows

$$
\begin{aligned}
& \frac{2 N+1}{N+1} \tilde{T}_{n+2}(x) \\
& =\frac{2 N+1}{N+1}\left((2 n+3)\left(1-x^{2}\right) \mathcal{D}_{\theta}(x) P_{n+1}(x)+\mathcal{D}_{r}(x)\left(n P_{n}(x)-(n+2) P_{n+2}(x)\right)\right) \\
& =N(2 n+3)\left(P_{N-1}(x)-P_{N+1}(x)\right) P_{n+1}(x)+(2 N+1) P_{N}\left(n P_{n}(x)-(n+2) P_{n+2}(x)\right) \\
& =\sum_{k}(2 k+1)(N(2 n+3)(\langle N-1 n+1 k\rangle-\langle N+1 n+1 k\rangle) \\
& \quad+(2 N+1)(n\langle N n k\rangle-(n+2)\langle N n+2 k\rangle)) P_{k}(x) \\
& =\sum_{k} c_{n k}^{N} \tilde{P}_{k}(x) \quad
\end{aligned}
$$

with

$$
\begin{aligned}
c_{n k}^{N}:=2 \sqrt{k+1 / 2} & (N(2 n+3)(\langle N-1 n+1 k\rangle-\langle N+1 n+1 k\rangle) \\
& +(2 N+1)(n\langle N n k\rangle-(n+2)\langle N n+2 k\rangle)) .
\end{aligned}
$$

Thus, $\left(\tilde{T}_{n+2}, g\right)_{\mathcal{H}}=0, n \in \mathbb{N}_{0}$ implies the recurrence relation

$$
\sigma_{n+N+2}+\sum_{k=0}^{2 N+1} \frac{c_{n, n+N-k+1}^{N}}{c_{n, n+N+2}^{N}} \sigma_{n+N-k+1}=0 .
$$


From (4.21) follows that $c_{n, n+N-2 k+1}^{N}=0$ for $k=0, \ldots, N$, so it suffices to determine

$$
d_{2 k}:=\lim _{n \rightarrow \infty} \frac{c_{n, n+N-2 k}^{N}}{c_{n, n+N+2}^{N}}, \quad k=0, \ldots, N .
$$

With (4.21) and (B.7), the coefficient $c_{n, n+N+2}^{N}$ can be rewritten as

$$
\begin{aligned}
c_{n, n+N+2}^{N}=2 \sqrt{N+n+5 / 2} & (-N(2 n+3)\langle N+1 n+1 N+n+2\rangle \\
& -(2 N+1)(n+2)\langle N n+2 N+n+2\rangle) \\
=-2 \sqrt{N+n+5 / 2} & \left(N(2 n+3) \frac{2 n+4}{2 n+3} \frac{2 N+1}{2 N+2}+(2 N+1)(n+2)\right) \\
\times & \langle N n+2 N+n+2\rangle,
\end{aligned}
$$

which becomes for large $n$ :

$$
c_{n, n+N+2}^{N} \sim-2 \frac{(2 N+1)^{2}}{N+1} n^{3 / 2}\langle N n+2 N+n+2\rangle .
$$

Here, $a_{n} \sim b_{n}, n \in \mathbb{N}$ means $\lim _{n \rightarrow \infty} a_{n} / b_{n}=1$. With (B.7) - (B.9) one obtains similarly for $c_{n, n+N-2 k}^{N}$ in the limit of large $n$ :

$$
c_{n, n+N-2 k}^{N} \sim-2 \frac{2 N+1}{k+1} n^{3 / 2}\langle N n N+n-2 k\rangle, \quad k=0, \ldots, N .
$$

Using the explicit representation (B.6) of $\langle N n k\rangle$ the ratio $\langle N n N+n-2 k\rangle /\langle N n+2 N+n+2\rangle$ can be asymptotically evaluated with the result:

$$
\lim _{n \rightarrow \infty} \frac{\langle N n N+n-2 k\rangle}{\langle N n+2 N+n+2\rangle}=\frac{\left(\begin{array}{c}
2 k \\
k
\end{array}\right)\left(\begin{array}{c}
2 N-2 k \\
N-k
\end{array}\right)}{\left(\begin{array}{c}
2 N \\
N
\end{array}\right)} .
$$

From $(4.24)-(4.26)$ follows then

$$
d_{2 k}=-\frac{N !(N+1) !}{(2 N+1) !} \frac{1}{k+1}\left(\begin{array}{c}
2 k \\
k
\end{array}\right)\left(\begin{array}{c}
2 N-2 k \\
N-k
\end{array}\right), \quad k=0, \ldots, N .
$$

(iv) According to Lemma 4.1, the polynomial $\tilde{Q}(X)$ corresponding to (4.22) now reads:

$$
\tilde{Q}(X)=X^{2 N+2}+\sum_{k=0}^{N} d_{2 k} X^{2 N-2 k} .
$$

From Eqs. (4.19) and (4.27) we immediately obtain $1+\sum_{k=0}^{N} d_{2 k}=0$ (cf. (Riordan 1979, Ch. 3, Ex. 11(a))) and by polynomial division, we arrive at $Q(X)=\frac{\tilde{Q}(X)}{X^{2}-1}=R\left(X^{2}\right)$, where

$$
R(Z):=Z^{N}+\sum_{k=1}^{N}\left(1+\sum_{j=0}^{k-1} d_{2 j}\right) Z^{N-k} \in \mathbb{R}[Z] .
$$

Since $d_{2 k}<0$ for $k=0, \ldots, N$, the coefficients $\kappa_{k}$ of $R(Z)=\sum_{k=0}^{N} \kappa_{k} Z^{k}$ satisfy $0<$ $\kappa_{0}<\kappa_{1}<\ldots<\kappa_{N}$ and Lemma 4.2 (b) yields $|z|<1$ for all zeroes of $R(Z)$ or $Q(X)$, respectively. Consequently, Lemma 4.1 guarantees the convergence of $\sum_{k=0}^{\infty}\left|\sigma_{k}\right|^{2}$ for an arbitrary choice of $\sigma_{0}, \ldots, \sigma_{N-1}$. Thus, we can conclude $\operatorname{dim} W_{D^{N}}^{\perp}=N$. 
4.2. Uniqueness of dipole-type direction fields. The following lemma generalizes Lemma 3.2 to the case of complete but not necessarily orthogonal function sets.

Lemma 4.4. Let $\left\{\psi_{n}: n \in \mathbb{N}_{0}\right\}$ be a complete system in a Hilbert space $\mathcal{H}$ and let $\left\{\chi_{n}: n \in \mathbb{N}_{0}\right\} \subset \mathcal{H}$. Let, furthermore, the inequality

$$
\left\|\sum_{n=0}^{N} a_{n}\left(\psi_{n}-\chi_{n}\right)\right\| \leq \Theta\left\|\sum_{n=0}^{N} a_{n} \psi_{n}\right\|
$$

hold for arbitrary $N \in \mathbb{N}_{0}, a_{n} \in \mathbb{C}, 0 \leq n \leq N$ and with $0<\Theta<1$ independent of $N$ and $a_{n}$. Then the system $\left\{\chi_{n}: n \in \mathbb{N}_{0}\right\}$ is complete in $\mathcal{H}$.

Proof. cf. Boas (1940) or de Sz. Nagy (1947).

TheOREM 4.5. Let $\mathbf{D}$ be a perturbed axisymmetric direction field which has either the form $\mathcal{D}_{r}(x)=2 x p(x), \mathcal{D}_{\theta}(x)=1$ or $\mathcal{D}_{r}(x)=2 x, \mathcal{D}_{\theta}(x)=\hat{p}(x)$ with $p, \hat{p} \in C^{3}([-1,1], \mathbb{R})$. Let the perturbations be small in the sense that

$$
\max _{[-1,1]} Q(x)+4|\|Q\||<\frac{3}{16}, \quad \max _{[-1,1]} \hat{Q}(x)+4|\|\hat{Q}\||<\frac{3}{8}
$$

with

$$
\begin{gathered}
\|Q\|:=\max \left\{\frac{4}{\sqrt{7}}\left\|Q^{\prime}\right\|, \frac{8}{3} \sqrt{\frac{10}{7}}\left\|Q^{\prime \prime}\right\|, 3 \sqrt{\frac{2}{5}}\left\|Q^{\prime \prime \prime}\right\|\right\} \\
Q(x):=x^{2}(1-p(x))^{2}, \quad \hat{Q}(x):=\left(1-x^{2}\right)(1-\hat{p}(x))^{2}
\end{gathered}
$$

and $\|\cdot\|$ denoting the norm in $\mathcal{H}=L^{2}((-1,1))$. Then there holds for the solution space $W_{\mathbf{D}}^{\perp}$ of Problem $\mathrm{P}_{3}^{a}$ :

$$
\operatorname{dim} W_{\mathbf{D}}^{\perp}=1
$$

Proof. (I) We treat the case of the perturbation $p(x)$ first and note in the case $\hat{p}(x)$ only the necessary changes.

(i) Setting $\psi_{n}(x):=\frac{1}{3(n+1)} \mathcal{T}_{n}(x)$ with $\mathcal{D}_{r}(x)=2 x, \mathcal{D}_{\theta}(x)=1$, and $\chi_{n}(x):=\frac{1}{3(n+1)} \mathcal{T}_{n}(x)$ with $\mathcal{D}_{r}(x)=2 x p(x), \mathcal{D}_{\theta}(x)=1$, we obtain with (2.26) and (A.4):

$$
\begin{aligned}
\psi_{0}(x) & =\frac{2}{3} P_{1}(x) \\
\psi_{n}(x) & =\frac{1}{3}\left(\left(P_{n+1}(x)-P_{n-1}(x)\right)+2 \frac{2 n+1}{n+1} x P_{n}(x)\right) \\
& =P_{n+1}(x)+\frac{1}{3} \frac{n-1}{n+1} P_{n-1}(x), n \in \mathbb{N}, \\
\psi_{n}(x) & -\chi_{n}(x)=\frac{2}{3} \frac{2 n+1}{n+1} x(1-p(x)) P_{n}(x)=: \frac{2}{3} \frac{2 n+1}{n+1} q(x) P_{n}(x), \quad n \in \mathbb{N}_{0} .
\end{aligned}
$$


(ii) With (A.2) the expression $\left\|\sum_{n=0}^{N} a_{n} \psi_{n}\right\|$, where $N \in \mathbb{N}$ and $a_{n} \in \mathbb{C}, n=0, \ldots, N$, can be evaluated as follows:

$$
\begin{aligned}
& \left\|\sum_{n=0}^{N} a_{n} \psi_{n}\right\|^{2}=\sum_{n, m=0}^{N} a_{n} a_{m}^{*}\left(\psi_{n}, \psi_{m}\right) \mathcal{H} \\
& =\frac{8}{27}\left|a_{0}\right|^{2}+\sum_{n=1}^{N} \frac{\left|a_{n}\right|^{2}}{n+3 / 2}+\frac{8}{81} \Re\left(a_{0} a_{2}^{*}\right)+\frac{2}{3} \sum_{n=1}^{N-2} \frac{n+1}{n+3} \frac{\Re\left(a_{n} a_{n+2}^{*}\right)}{n+3 / 2} \\
& \quad+\frac{1}{9} \sum_{n=2}^{N}\left(\frac{n-1}{n+1}\right)^{2} \frac{\left|a_{n}\right|^{2}}{n-1 / 2} .
\end{aligned}
$$

Here, $\Re($.$) denotes the real part of (.). With the estimate$

$$
\begin{aligned}
2 \sum_{n=1}^{N-2} \frac{n+1}{n+3} \frac{\Re\left(a_{n} a_{n+2}^{*}\right)}{n+3 / 2} & \leq \sum_{n=1}^{N-2} \frac{n+1}{n+3} \frac{\left|a_{n}\right|^{2}}{n+3 / 2}+\sum_{n=3}^{N} \frac{n-1}{n+1} \frac{\left|a_{n}\right|^{2}}{n-1 / 2} \\
& \leq \sum_{n=1}^{N} \frac{\left|a_{n}\right|^{2}}{n+3 / 2}+\sum_{n=3}^{N} \frac{\left|a_{n}\right|^{2}}{n+3 / 2} \leq 2 \sum_{n=1}^{N} \frac{\left|a_{n}\right|^{2}}{n+3 / 2}
\end{aligned}
$$

the expression $\left\|\sum_{n=0}^{N} a_{n} \psi_{n}\right\|^{2}$ can thus be estimated from below:

$$
\begin{aligned}
\left\|\sum_{n=0}^{N} a_{n} \psi_{n}\right\|^{2} & \geq \frac{8}{27}\left|a_{0}\right|^{2}+\sum_{n=1}^{N} \frac{\left|a_{n}\right|^{2}}{n+3 / 2}-\frac{4}{81}\left(\left|a_{0}\right|^{2}+\left|a_{2}\right|^{2}\right)-\frac{2}{3} \sum_{n=1}^{N} \frac{\left|a_{n}\right|^{2}}{n+3 / 2} \\
& \geq \frac{10}{27}\left|a_{0}\right|^{2}+\frac{1}{3} \sum_{n=1}^{N} \frac{\left|a_{n}\right|^{2}}{n+3 / 2} \geq \frac{1}{3} \sum_{n=0}^{N} \frac{\left|a_{n}\right|^{2}}{n+3 / 2} .
\end{aligned}
$$

(iii) The perturbed expression $\left\|\sum_{n=0}^{N} a_{n}\left(\psi_{n}-\chi_{n}\right)\right\|^{2}$ is decomposed as follows:

$$
\begin{aligned}
\left\|\sum_{n=0}^{N} a_{n}\left(\psi_{n}-\chi_{n}\right)\right\|^{2}= & \frac{4}{9} \sum_{m, n=0}^{N} \frac{2 m+1}{m+1} \frac{2 n+1}{n+1} a_{m} a_{n}^{*}\left(q P_{m}, q P_{n}\right)_{\mathcal{H}} \\
= & \frac{4}{9} \sum_{n=0}^{N}\left(\frac{2 n+1}{n+1}\right)^{2}\left|a_{n}\right|^{2} \int_{-1}^{1} q^{2}(x)\left(P_{n}(x)\right)^{2} d x \\
& +\frac{4}{9} \sum_{\substack{m, n=0 \\
m \neq n}}^{N} \frac{2 m+1}{m+1} \frac{2 n+1}{n+1} a_{m} a_{n}^{*} \int_{-1}^{1} q^{2}(x) P_{m}(x) P_{n}(x) d x
\end{aligned}
$$

with $q(x)=x(1-p(x))$. In (iv) we prove the estimate

$$
\left|\int_{-1}^{1} q^{2}(x) P_{m}(x) P_{n}(x) d x\right| \leq \frac{4}{3} \frac{|\|Q\||}{\sqrt{(2 m+1)(2 n+1)}} \frac{1}{|m-n|^{3 / 2}}, \quad m, n \in \mathbb{N}_{0}, m \neq n
$$


with $|\|Q\||$ explained in (4.30). Furthermore, we have with $\sum_{\nu=1}^{\infty} \frac{1}{\nu^{3 / 2}} \leq 3$ analogously to the estimate (3.8) in Sec. 3:

$$
\begin{aligned}
& \sum_{\substack{m, n=0 \\
m \neq n}}^{N} \frac{\left|a_{m}\right|\left|a_{n}\right|}{|m-n|^{3 / 2}}=\sum_{\substack{m, n=0 \\
m \neq n}}^{N}\left|a_{m}\right|\left|a_{n}\right| \sum_{\nu=-\infty}^{\infty} \frac{1}{|\nu|^{3 / 2}} \cdot \frac{1}{2 \pi} \int_{-\pi}^{\pi} e^{i(m-n-\nu) x} d x \\
& =\frac{1}{2 \pi} \int_{-\pi}^{\pi} \sum_{m, n=0}^{N}\left|a_{m}\right|\left|a_{n}\right| e^{i(m-n) x} \sum_{\substack{\nu=-\infty \\
\nu \neq 0}}^{\infty} \frac{e^{-i \nu x}}{|\nu|^{3 / 2}} d x \\
& \leq \frac{1}{2 \pi} \int_{-\pi}^{\pi}\left|\sum_{n=0}^{N}\right| a_{n}\left|e^{i n x}\right|^{2} \cdot 2 \sum_{\nu=1}^{\infty} \frac{1}{\nu^{3 / 2}} d x \leq 6 \sum_{n=0}^{N}\left|a_{n}\right|^{2} .
\end{aligned}
$$

Thus we can estimate $\left\|\sum_{n=0}^{N} a_{n}\left(\psi_{n}-\chi_{n}\right)\right\|^{2}$ :

$$
\begin{aligned}
& \left\|\sum_{n=0}^{N} a_{n}\left(\psi_{n}-\chi_{n}\right)\right\|^{2} \leq \frac{4}{9} \max _{[-1,1]} Q(x) \sum_{n=0}^{N}\left(\frac{2 n+1}{n+1}\right)^{2} \frac{\left|a_{n}\right|^{2}}{n+1 / 2} \\
& \quad+\frac{16}{27}|\|Q\|| \sum_{\substack{m, n=0 \\
m \neq n}}^{N} \frac{2 m+1}{m+1} \frac{2 n+1}{n+1} \frac{\left|a_{m}\right|\left|a_{n}\right|}{\sqrt{(2 m+1)(2 n+1)}} \frac{1}{|m-n|^{3 / 2}} \\
& \leq \frac{16}{9} \max _{[-1,1]} Q(x) \sum_{n=0}^{N} \frac{\left|a_{n}\right|^{2}}{n+3 / 2}+\frac{32}{27}|\|Q\|| \sum_{\substack{m, n=0 \\
m \neq n}}^{N} \frac{\left|a_{m}\right|}{\sqrt{m+3 / 2}} \frac{\left|a_{n}\right|}{\sqrt{n+3 / 2}} \frac{1}{|m-n|^{3 / 2}} \\
& \leq \frac{16}{9}\left(\max _{[-1,1]} Q(x)+4|\|Q\||\right) \sum_{n=0}^{N} \frac{\left|a_{n}\right|^{2}}{n+3 / 2} .
\end{aligned}
$$

(iv) We prove here inequality (4.33). Let us first consider the expression $\int_{-1}^{1} Q(x) P_{\nu}(x) d x$ with $\nu \geq 3$. Repeated partial integration and use of Eq. (A.6) yields:

$$
\begin{aligned}
\int_{-1}^{1} Q(x) P_{\nu}(x) d x= & -\frac{1}{4} \frac{1}{2 \nu+1} \int_{-1}^{1} Q^{\prime \prime \prime}(x)\left(\frac{P_{\nu+3}}{(\nu+5 / 2)(\nu+3 / 2)}-\frac{3 P_{\nu+1}}{(\nu+5 / 2)(\nu-1 / 2)}\right. \\
& \left.+\frac{3 P_{\nu-1}}{(\nu+3 / 2)(\nu-3 / 2)}-\frac{P_{\nu-3}}{(\nu-1 / 2)(\nu-3 / 2)}\right) d x
\end{aligned}
$$

Thus, with Cauchy-Schwarz and (A.2):

$$
\begin{aligned}
& \left|\int_{-1}^{1} Q(x) P_{\nu}(x) d x\right| \\
& \leq \frac{1}{4} \frac{1}{2 \nu+1} \frac{1}{\nu^{5 / 2}}\left[\frac{\nu^{5}}{(\nu+7 / 2)(\nu+5 / 2)^{2}(\nu+3 / 2)^{2}}+\frac{9 \nu^{5}}{(\nu+5 / 2)^{2}(\nu+3 / 2)(\nu-1 / 2)^{2}}\right. \\
& \left.\quad+\frac{9 \nu^{5}}{(\nu+3 / 2)^{2}(\nu-1 / 2)(\nu-3 / 2)^{2}}+\frac{\nu^{5}}{(\nu-1 / 2)^{2}(\nu-3 / 2)^{2}(\nu-5 / 2)}\right]^{1 / 2}\left\|Q^{\prime \prime \prime}\right\| \\
& \leq 3 \sqrt{\frac{2}{5}} \frac{1}{2 \nu+1} \frac{1}{\nu^{5 / 2}}\left\|Q^{\prime \prime \prime}\right\|, \quad \nu \geq 3 .
\end{aligned}
$$


In the last estimate we made use of the fact that the bracket takes its maximum for $\nu=3$. Similarly, we obtain for $\nu=1$ and 2 :

$$
\begin{gathered}
\left|\int_{-1}^{1} Q(x) P_{1}(x) d x\right| \leq \frac{4}{\sqrt{7}} \frac{1}{3}\left\|Q^{\prime}\right\| \\
\left|\int_{-1}^{1} Q(x) P_{2}(x) d x\right| \leq \frac{8}{3} \sqrt{\frac{10}{7}} \frac{1}{5} \frac{1}{2^{5 / 2}}\left\|Q^{\prime \prime}\right\| .
\end{gathered}
$$

Summarizing $(4.35)-(4.37)$, we have

$$
\left|\int_{-1}^{1} Q(x) P_{\nu}(x) d x\right| \leq \frac{1}{2 \nu+1} \frac{1}{\nu^{5 / 2}}|\|Q\||, \quad \nu \in \mathbb{N} .
$$

Next, we decompose $P_{m}(x) P_{n}(x)$ according to (B.3), make use of the properties of the coefficients $\langle m n \nu\rangle$, in particular of (4.21), and of

$$
\langle m n \nu\rangle \leq \frac{1}{\sqrt{(2 m+1)(2 n+1)}}
$$

and obtain with (4.38):

$$
\begin{gathered}
\left|\int_{-1}^{1} q^{2}(x) P_{m}(x) P_{n}(x) d x\right| \leq \sum_{\nu}(2 \nu+1)\langle m n \nu\rangle\left|\int_{-1}^{1} Q(x) P_{\nu}(x) d x\right| \\
\leq \frac{|\|Q\||}{\sqrt{(2 m+1)(2 n+1)}} \sum_{\substack{\nu=|m-n| \\
\nu-|m-n| \in 2 \mathbb{Z}}}^{m+n} \frac{1}{\nu^{5 / 2}} \leq \frac{4}{3} \frac{|\|Q\||}{\sqrt{(2 m+1)(2 n+1)}} \frac{1}{|m-n|^{3 / 2}},
\end{gathered}
$$

which proves (4.33).

In the last estimate we made use of

$$
\sum_{\substack{n=n_{0} \\ n-n_{0} \in 2 \mathbb{Z}}}^{\infty} \frac{1}{n^{\alpha}} \leq \frac{1}{n_{0}^{\alpha}}+\frac{1}{2} \int_{n_{0}}^{\infty} \frac{1}{x^{\alpha}} d x=\frac{1}{n_{0}^{\alpha-1}}\left(\frac{1}{n_{0}}+\frac{1}{2(\alpha-1)}\right) \leq \frac{1}{n_{0}^{\alpha-1}}\left(1+\frac{1}{2(\alpha-1)}\right)
$$

which is valid for $n \in \mathbb{N}$ and any real $\alpha>1$. Inequality (4.39) follows from (B.3) with $x=1$, which implies $\langle m n \nu\rangle \leq \frac{1}{2 \nu+1}$ and the total symmetry of $\langle m n \nu\rangle$, which implies, moreover,

$$
\langle m n \nu\rangle \leq \min \left\{\frac{1}{2 m+1}, \frac{1}{2 n+1}, \frac{1}{2 \nu+1}\right\} .
$$

(v) We now prove the statement of the theorem. From (4.32), (4.34), and the first condition in (4.29) follows that the systems $\left\{\psi_{n}: n \in \mathbb{N}_{0}\right\}$ and $\left\{\chi_{n}: n \in \mathbb{N}_{0}\right\}$ satisfy condition (4.28) in Lemma 4.4. Since $P_{0} \perp \psi_{n}, n \in \mathbb{N}_{0}$, condition (4.28) holds with the same $\Theta$ also for the enlarged systems $\left\{P_{0}, \psi_{n}: n \in \mathbb{N}_{0}\right\}$ and $\left\{P_{0}, \chi_{n}: n \in \mathbb{N}_{0}\right\}$. As the first system is complete, so is the second. On the other hand, there is $P_{0} \notin \operatorname{Span}\left\{\chi_{n}\right.$ : $\left.n \in \mathbb{N}_{0}\right\}$ since the assumption $P_{0}=\sum_{n=0}^{\infty} b_{n} \chi_{n}$ with $b_{n} \in \mathbb{C}$ leads, with (4.28), to the contradiction

$$
\begin{aligned}
\left\|\sum_{n=0}^{\infty} b_{n} \psi_{n}\right\|^{2}+\left\|P_{0}\right\|^{2} & =\left\|\sum_{n=0}^{\infty} b_{n} \psi_{n}-P_{0}\right\|^{2}=\left\|\sum_{n=0}^{\infty} b_{n}\left(\psi_{n}-\chi_{n}\right)\right\|^{2} \\
& \leq \Theta\left\|\sum_{n=0}^{\infty} b_{n} \psi_{n}\right\|^{2}, \quad 0<\Theta<1
\end{aligned}
$$


So, we have $\operatorname{dim}\left\{\chi_{n}: n \in \mathbb{N}_{0}\right\}^{\perp}=1$, which is according to Corollary 2.5 the statement.

(II) In the case that the dipole field is perturbed by $\hat{p}(x)$, we define the $\psi_{n}(x)$ as before and $\chi_{n}(x):=\frac{1}{3(n+1)} \mathcal{T}_{n}(x)$ with $\mathcal{D}_{r}(x)=2 x$ and $\mathcal{D}_{\theta}(x)=\hat{p}(x) . \psi_{n}-\chi_{n}$ with $($ A.5) and (A.1) now takes the form

$$
\begin{aligned}
\psi_{0}(x)-\chi_{0}(x) & =0 \\
\psi_{n}(x)-\chi_{n}(x) & =\frac{1}{3}(1-\hat{p}(x))\left(P_{n+1}(x)-P_{n-1}(x)\right) \\
& =\frac{1}{3} \frac{2 n+1}{n(n+1)} \sqrt{1-x^{2}}(\hat{p}(x)-1) P_{n}^{1}(x) \\
& =: \frac{1}{3} \frac{2 n+1}{n(n+1)} \hat{q}(x) P_{n}^{1}(x), \quad n \in \mathbb{N} .
\end{aligned}
$$

The estimate (ii) remains as before and (iii) now becomes:

$$
\begin{aligned}
\left\|\sum_{n=0}^{N} a_{n}\left(\psi_{n}-\chi_{n}\right)\right\|^{2} & =\frac{1}{9} \sum_{n=1}^{N}\left(\frac{2 n+1}{n(n+1)}\right)^{2}\left|a_{n}\right|^{2} \int_{-1}^{1} \hat{q}^{2}(x)\left(P_{n}^{1}(x)\right)^{2} d x \\
& +\frac{1}{9} \sum_{\substack{m, n=1 \\
m \neq n}}^{N} \frac{2 m+1}{m(m+1)} \frac{2 n+1}{n(n+1)} a_{m} a_{n}^{*} \int_{-1}^{1} \hat{q}^{2}(x) P_{m}^{1}(x) P_{n}^{1}(x) d x .
\end{aligned}
$$

Instead of (4.33) we now use the estimate

$$
\left|\int_{-1}^{1} \hat{q}^{2}(x) P_{m}^{1}(x) P_{n}^{1}(x) d x\right| \leq \frac{4}{3} \sqrt{\frac{m(m+1) n(n+1)}{(2 m+1)(2 n+1)}} \frac{|\|\hat{Q}\||}{|m-n|^{3 / 2}}, \quad m, n \in \mathbb{N}_{0}, m \neq n .
$$

Thus, we can estimate analogously to (iii):

$$
\begin{aligned}
\left\|\sum_{n=0}^{N} a_{n}\left(\psi_{n}-\chi_{n}\right)\right\|^{2} \leq & \frac{1}{9} \max _{[-1,1]} \hat{Q}(x) \sum_{n=1}^{N}\left(\frac{2 n+1}{n(n+1)}\right)^{2} \frac{n(n+1)}{n+1 / 2}\left|a_{n}\right|^{2} \\
& \quad+\frac{4}{27}|\|\hat{Q}\|| \sum_{\substack{n, n=1 \\
m \neq n}}^{N} \sqrt{\frac{(2 m+1)(2 n+1)}{m(m+1) n(n+1)}} \frac{\left|a_{m}\right|\left|a_{n}\right|}{|m-n|^{3 / 2}} \\
\leq & \frac{8}{9} \max _{[-1,1]} \hat{Q}(x) \sum_{n=1}^{N} \frac{\left|a_{n}\right|^{2}}{n+3 / 2}+\frac{16}{27}|\|\hat{Q}\|| \sum_{\substack{m, n=1 \\
m \neq n}}^{N} \frac{\left|a_{m}\right|}{\sqrt{m+3 / 2}} \frac{\left|a_{n}\right|}{\sqrt{n+3 / 2}} \frac{1}{|m-n|^{3 / 2}} \\
\leq & \frac{8}{9}\left(\max _{[-1,1]} \hat{Q}(x)+4|\|\hat{Q}\||\right) \sum_{n=1}^{N} \frac{\left|a_{n}\right|^{2}}{n+3 / 2} .
\end{aligned}
$$

In the derivation of inequality (4.40), the only difference to (iv) is that the expansion (B.3) is replaced by (B.4). Moreover, the estimate

$$
\frac{1}{2}(\nu(\nu+1)-m(m+1)-n(n+1)) \leq \sqrt{m(m+1) n(n+1)} \quad \text { if } \quad|m-n| \leq \nu \leq m+n
$$

has been used.

The rest of the proof in (I) applies without changes to the present situation. 
REMARK 4.6. Let us compare Theorems 4.3 and 4.5 to the results of Hulot et al. (1997) in the case of axisymmetry. These authors derived an upper bound on the dimension of the solution space equal to $l-1$ with $l$ being the number of loci with $\mathcal{D}_{\theta}=0$ (poles). This implies uniqueness in the case that there are not more than two poles on $S^{2}$. No smallness condition is required. Theorem 4.3 demonstrates that in terms of poles, this bound cannot be improved. Theorem 4.5, on the other hand, guarantees uniqueness for all direction fields which are close to $\mathbf{D}_{1}$ in the sense of condition (4.29). Note that the $\hat{p}$-perturbation seems to allow for direction fields with multiple poles close to $\theta=0$ or $\pi$. However, we were neither able to find an explicit example of such a field nor to prove the contrary. In the first case the statement in Theorem 4.5 would not be implied by the result of Hulot et al. (1997), and the number of poles would in general overestimate the dimension of the solution space (cf. Sec. 4.4).

4.3. Additional constraints and uniqueness. The following theorem and remark present possibilities to enforce uniqueness in the problem $\mathrm{P}_{3}^{a}$ by imposing additional constraints. The last remark deals with the so-called signed direction problem.

Theorem 4.7. Let $N \in \mathbb{N}$ and $\mathbf{D}_{N}$ be the axisymmetric $2^{N}$ pole field $\mathcal{D}_{r}^{N}(x)=$ $(N+1) P_{N}(x), \mathcal{D}_{\theta}^{N}(x)=P_{N}^{\prime}(x)$. Then the modified problem $\mathrm{P}_{3}^{a}$ where $\Psi$ has additionally to satisfy

$$
|\Psi|=O\left(|\mathbf{x}|^{-(N+1)}\right) \quad \text { for }|\mathbf{x}| \rightarrow \infty
$$

is uniquely solvable.

Proof. Let $g=\sum_{k=0}^{\infty} \tau_{k} P_{k}$ and let us write the $\left(\mathcal{T}_{n}\right)_{n \in \mathbb{N}_{0}}$ with $\mathbf{D}=\mathbf{D}_{N}$ and with help of (A.1) and (A.5) in the form

$$
\begin{aligned}
& \mathcal{T}_{0}=(N+1) P_{N}, \\
& \mathcal{T}_{n}=(2 n+1)\left((N+1) P_{N} P_{n}-\frac{1}{n} P_{N}^{1} P_{n}^{1}\right), \quad n \in \mathbb{N} .
\end{aligned}
$$

Using (B.3) and (B.4), the condition $\left(\mathcal{T}_{n}, g\right)_{\mathcal{H}}=0$ then implies $\tau_{N}=0$ and

$$
\sum_{k=N-n}^{N+n}\left(2(N+1)+\frac{1}{n} \Gamma_{N n k}\right)\langle N n k\rangle \tau_{k}=0, \quad n \in \mathbb{N}
$$

with the abbreviation $\Gamma_{N n k}:=k(k+1)-N(N+1)-n(n+1)$. The condition (4.41) yields $b_{n}=0, n=1, \ldots, N-1$ in the expansion (4.13) of $\Psi$, and this implies with (4.14) and (4.42):

$$
\sum_{k=N-n}^{N+n}\langle N n k\rangle \tau_{k}=0, \quad \sum_{k=N-n}^{N+n} \Gamma_{N n k}\langle N n k\rangle \tau_{k}=0, \quad n=1, \ldots, N-1 .
$$

Starting with $n=1$, which yiclds $\tau_{N-1}=\tau_{N+1}=0$, Eqs. (4.43) can now successively be evaluated with the result

$$
\tau_{1}=\tau_{2}=\ldots=\tau_{2 N-1}=0 .
$$

Observe here that $\langle N n N \pm n\rangle \neq 0$ and that $\Gamma_{N n N+n} \neq \Gamma_{N n N-n}$. Equation (4.42) with $n=N$ yields then $\tau_{2 N}=0$, and for all $k>2 N$ there is $\tau_{k}=0$ as is obvious from 
the recurrence relation (4.3). Therefore, the only coefficient $\neq 0$ is $\tau_{0}$, which proves our claim.

REMARK 4.8. Another possibility to enforce uniqueness is to allow only solutions which are represented by finite sums of Legendre polynomials: Let $N \in \mathbb{N}, \mathcal{D}_{r}(x)=$ $(N+1) P_{N}(x), \mathcal{D}_{\theta}(x)=P_{N}^{\prime}(x)$ as in Theorem 4.3 , and $g \in W_{a}^{\perp}$ with $g(x) \neq 0$ almost everywhere. Then the solution $\Psi$ in Problem $\mathrm{P}_{3}^{a}$ is of the form

$$
\Psi=c r^{-(N+1)} \tilde{P}_{N}(\cos \theta) \quad \text { or } \quad \Psi=\sum_{k=1}^{\infty} c_{k} r^{-(k+1)} \tilde{P}_{k}(\cos \theta)
$$

with infinitely many $c_{k} \neq 0$.

Proof. Let $n_{0} \in \mathbb{N}$ and $\Psi=\sum_{k=1}^{n_{0}} c_{k} r^{-(k+1)} \tilde{P}_{k}(\cos \theta)$ be any axisymmetric solution of Problem (2.11). Then the traces of $\partial_{r} \Psi, \partial_{\theta} \Psi$ on $S^{2}$ are given by (cf. Corollary 2.5)

$$
\begin{gathered}
(N+1) g(\cos \theta) P_{N}(\cos \theta)=-\sum_{k=1}^{n_{0}}(k+1) c_{k} \tilde{P}_{k}(\cos \theta), \\
g(\cos \theta) P_{N}^{\prime}(\cos \theta) \sin \theta=-\sum_{k=1}^{n_{0}} c_{k} P_{k}^{\prime}(\cos \theta) \sin \theta
\end{gathered}
$$

respectively. Therefore, we have

$$
\sum_{k=1}^{n_{0}} c_{k}\left((N+1) P_{N}(x) \tilde{P}_{k}^{\prime}(x)-(k+1) P_{N}^{\prime}(x) \tilde{P}_{k}(x)\right)=0, \quad x=\cos \theta .
$$

Obviously, the term in parentheses on the left-hand side vanishes if $k=N$ and is a polynomial of degree $k+N-1$ if $k \neq N$. Consequently, there is $c_{k}=0$ for $k \neq N$.

REMARK 4.9. The signed direction problem prescribes the direction including the sign on $S^{2}$; i.e., the function $f$ in $\mathrm{P}_{3}$, resp. $g$ in $\mathrm{P}_{3}^{a}$ is not allowed to change the sign. The quadrupole example above demonstrates that, in general, the non-uniqueness of the unsigned direction problem holds likewise for the signed problem. Note that the solution $g$ depends on the parameters $\sigma_{0}$ and $\sigma_{1}$; i.e., $g=g\left[\sigma_{0}, \sigma_{1}\right] . g\left[\sigma_{0}, 0\right]$ is obviously a solution of the signed problem, whereas $g\left[0, \sigma_{1}\right]$ is not since all $\tilde{P}_{2 k+1}, k \in \mathbb{N}_{0}$, and hence $g\left[0, \sigma_{1}\right]$ are odd functions. Whether $g\left[\sigma_{0}, \sigma_{1}\right]$ is a solution of the signed problem depends on the ratio of $\sigma_{0}$ and $\sigma_{1}$. This demonstrates that the restriction of the direction problem to the signed variant does not guarantee uniqueness. Moreover, the set of solutions of the signed problem is no longer a linear space.

4.4. Solution space and rotation number. For 3-dimensional axisymmetric direction fields a rotation number can be introduced quite analogously to the 2-dimensional case. This subsection provides some evidence that this rotation number determines the dimension of the solution space.

Let $\mathbf{D} \in C^{1}\left(S^{2}, \mathbb{R}\right)$ be a nowhere vanishing axisymmetric direction field with representation $\mathbf{D}(\theta)=D_{r}(\theta) \mathbf{e}_{r}(\theta)+D_{\theta}(\theta) \mathbf{e}_{\theta}(\theta)$. The quantity

$$
\phi(\theta):=\int_{0}^{\theta} \frac{d}{d \tilde{\theta}}\left(\arctan \frac{D_{\theta}(\tilde{\theta})}{D_{r}(\tilde{\theta})}\right) d \tilde{\theta}=\int_{0}^{\theta} \frac{D_{r}(\tilde{\theta}) \frac{d}{d \tilde{\theta}} D_{\theta}(\tilde{\theta})-D_{\theta}(\tilde{\theta}) \frac{d}{d \tilde{\theta}} D_{r}(\tilde{\theta})}{D_{r}^{2}(\tilde{\theta})+D_{\theta}^{2}(\tilde{\theta})} d \tilde{\theta}
$$


measures the rotation angle of $\mathbf{D}(\theta)$ with respect to the normal $\mathbf{e}_{r}(\theta)$. A suitable rotation number $\rho$, measuring the number of turns of $\mathbf{D}$ in the interval $0<\theta<\pi$, is given by

$$
\rho:=\frac{1}{2}+\frac{\phi(\pi)}{2 \pi}
$$

Note that the rotation number refers to a fixed frame which explains the $1 / 2$ in Eq. (4.45). Note, furthermore, that $2 \rho \in \mathbb{Z}$ since $D_{\theta}=0$ at $\theta=0$ and $\pi$.

In the following we investigate three simple direction fields, viz:

$$
\mathbf{D}_{M N}^{\lambda}(\theta):=\mathbf{D}_{M}(\theta)+\lambda \mathbf{D}_{N}(\theta), \quad(M, N)=(1,2),(1,3),(2,3), \quad \lambda \in[0, \infty)
$$

with

$$
\mathbf{D}_{N}(\theta)=(N+1) P_{N}(\cos \theta) \mathbf{e}_{r}+P_{N}^{\prime}(\cos \theta) \sin \theta \mathbf{e}_{\theta},
$$

and $\mathbf{D}_{M N}^{\infty}:=\mathbf{D}_{N}$. Rotation angle and rotation number are determined from (4.44) and (4.45), respectively. Plots of $\phi(\theta)$ for various values of $\lambda>0$ are shown for $\mathbf{D}_{12}^{\lambda}$ and $\mathbf{D}_{13}^{\lambda}$ in Figs. 2 and 3, respectively. The case $\lambda=0$, where $\mathbf{D}_{12}^{0}=\mathbf{D}_{13}^{0}=\mathbf{D}_{1}$, is shown in Fig. 1 .

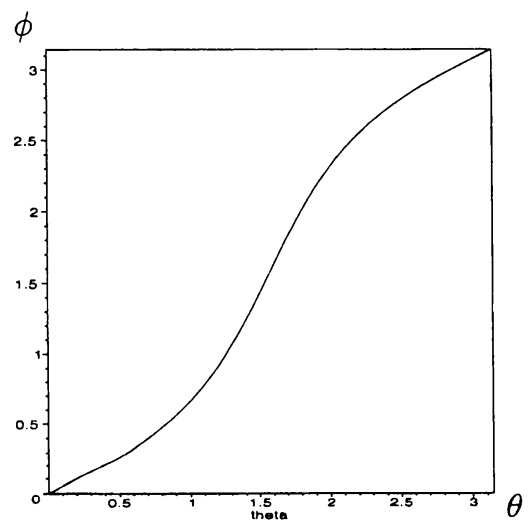

FIG. 1. Rotation angle $\phi$ versus $\theta$ of the direction field $\mathbf{D}_{1}$

Figure 1 and the right plots of Figs. 2 and 3 refer to rotation functions $\phi(\theta)$ of pure multipole fields, whereas the other plots demonstrate a jump of the rotation number at the critical value $\lambda=2 / 3$. Similar features are observed for $\mathbf{D}_{23}^{\lambda}$ (not shown). For the rotation number $\rho_{M N}$ of $\mathbf{D}_{M N}^{\lambda}$ as a function of $\lambda$, we find:

$$
\rho_{12}=\left\{\begin{array}{ll}
1 & \text { for } \lambda<\frac{2}{3} \\
\frac{3}{2} & \text { for } \lambda>\frac{2}{3}
\end{array} \quad, \quad \rho_{13}=\left\{\begin{array}{ll}
1 & \text { for } \lambda<\frac{2}{3} \\
2 & \text { for } \lambda>\frac{2}{3}
\end{array} \quad, \quad \rho_{23}= \begin{cases}\frac{3}{2} & \text { for } \lambda<\frac{3}{4} \\
2 & \text { for } \lambda>\frac{3}{4}\end{cases}\right.\right.
$$

On the other side, the dimension of the solution space $\operatorname{dim} W_{\mathbf{D}}^{\perp}$ is determined for $\mathbf{D}=$ $\mathbf{D}_{M N}^{\lambda}$. In all three cases and for all $\lambda \in[0, \infty]$, there is $\operatorname{dim} W_{\mathbf{D}}^{\perp} \geq 1$ since $g \equiv 1$ is obviously a solution of the problem $\mathrm{P}_{3}^{a}$. In order to find further solutions we proceed as in Sec. 4.1; i.e., formal solutions are given by series expansions whose coefficients obey recurrence relations of the type (4.3). These coefficients still depend on $\lambda$ and the convergence of the series has to be investigated in dependence on $\lambda$. This has been 

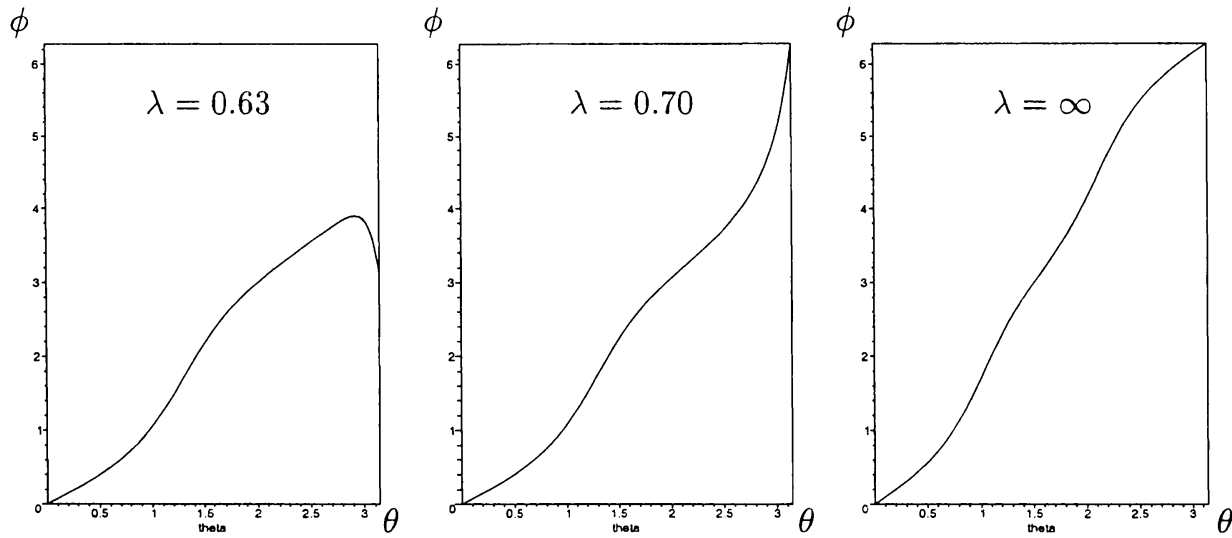

FIG. 2. Rotation angle $\phi$ versus $\theta$ of the direction field $\mathbf{D}_{12}^{\lambda}$ for three different values of the parameter $\lambda$. Observe the jump in the rotation number at $\lambda=\frac{2}{3}$.
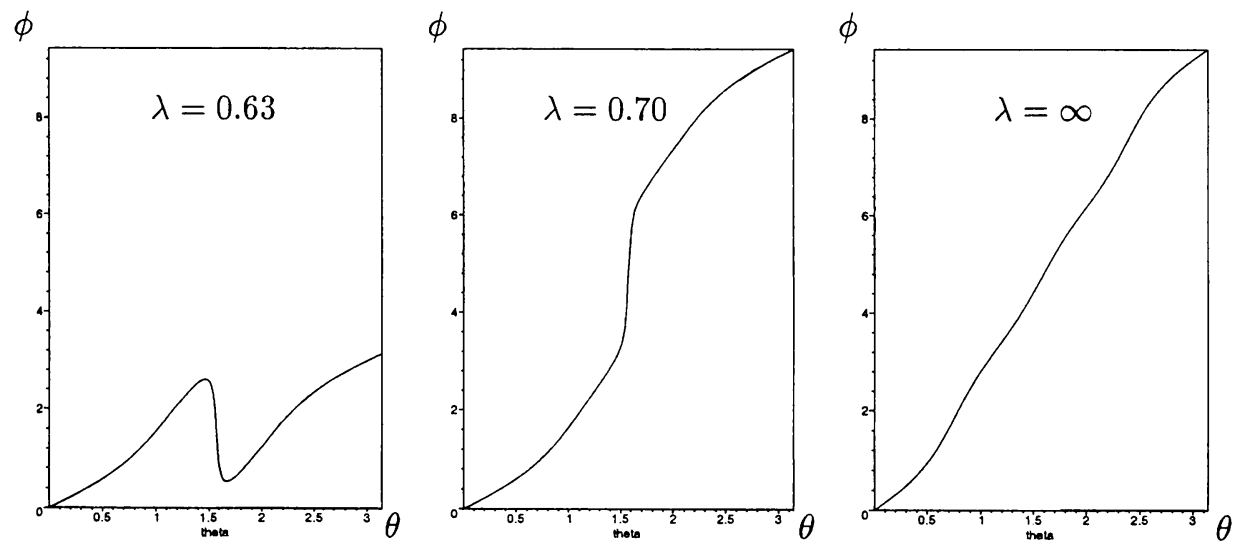

FIG. 3. The same as Fig. 2 for the direction field $\mathbf{D}_{13}^{\lambda}$

done numerically for all three direction fields and for a variety of $\lambda$ values. In the case of $\mathbf{D}_{12}^{\lambda}$ there is just one series, and we find convergence for all tested values above the critical value $\lambda=2 / 3$ and divergence below. The same critical $\lambda$ value appears for $\mathbf{D}_{13}^{\lambda}$. However, there are now two linear independent series; we find both converging above the critical value and none converging below.

In the case of $\mathbf{D}_{23}^{\lambda}$ the recurrence relations define a series depending on two parameters, $\sigma_{1}$ and $\sigma_{2}$. For $\lambda>\frac{3}{4}$ the series converges for all values of $\sigma_{1}$ and $\sigma_{2}$. For $\lambda<\frac{3}{4}$ the situation is more complicated: A first rough test did not reveal any converging series. In a second step, we investigated finite sums $S(\lambda, v, m):=\sum_{k=1}^{m}\left|\sigma_{k}\right|^{2}$ with $v$ being the ratio $\sigma_{1} / \sigma_{2}$. Varying $v$ for fixed $m$ and $\lambda<\frac{3}{4}$ we found a sharp minimum of $S(\lambda, v, m)$ at some value $v=v_{0}(\lambda, m)$. Enlarging $m$ we found, furthermore, $v_{0}(\lambda, m)$ converging to 
some $v_{0}(\lambda)$. We interpret these numerical findings as an indication of a converging series for $v_{0}(\lambda)$ with $\lambda<\frac{3}{4}$.

Summarizing these results, we have:

$$
\begin{gathered}
\operatorname{dim} W_{\mathbf{D}_{12}^{\lambda}}^{\perp}=\left\{\begin{array}{ll}
1 & \text { for } \lambda<\frac{2}{3} \\
2 & \text { for } \lambda>\frac{2}{3}
\end{array}, \operatorname{dim} W_{\mathbf{D}_{13}^{\lambda}}^{\perp}=\left\{\begin{array}{ll}
1 & \text { for } \lambda<\frac{2}{3} \\
3 & \text { for } \lambda>\frac{2}{3}
\end{array},\right.\right. \\
\operatorname{dim} W_{\mathbf{D}_{23}^{\lambda}}^{\perp}=\left\{\begin{array}{ll}
2 & \text { for } \lambda<\frac{3}{4} \\
3 & \text { for } \lambda>\frac{3}{4}
\end{array} .\right.
\end{gathered}
$$

A comparison of (4.46) and (4.47) suggests the following conjecture:

ConjeCture 4.10. Let $\mathbf{D}: S^{2} \rightarrow \mathbb{R}^{3}$ be a nowhere vanishing axisymmetric direction field with rotation number $\rho$. Then, the dimension of the solution space $\operatorname{dim} W_{\mathbf{D}}^{\perp}$ of problem $\mathrm{P}_{3}^{a}$ is given by

$$
\operatorname{dim} W_{\mathbf{D}}^{\perp}=\left\{\begin{array}{cc}
2 \rho-1 & \text { if } \rho \geq 1 \\
0 & \text { otherwise }
\end{array}\right.
$$

A. Appendix. This appendix collects some definitions, conventions and relations about Legendre polynomials and spherical harmonics which are used in the main part of the paper (cf. Abramowitz \& Stegun 1972).

The Legendre polynomials $P_{n}$ are defined on the interval $(-1,1)$ by

$$
P_{n}(x):=\frac{1}{2^{n} n !} \frac{d^{n}}{d x^{n}}\left(\left(x^{2}-1\right)^{n}\right), \quad n \in \mathbb{N}_{0},
$$

and the associated Legendre functions $P_{n}^{m}$ by

$$
P_{n}^{m}(x):=\left(1-x^{2}\right)^{m / 2} \frac{d^{m}}{d x^{m}} P_{n}(x), \quad n \in \mathbb{N}_{0}, m=0, \ldots, n .
$$

They satisfy the orthogonality condition

$$
\int_{-1}^{1} P_{n}^{m}(x) P_{n^{\prime}}^{m} d x=\frac{2}{2 n+1} \frac{(n+m) !}{(n-m) !} \delta_{n n^{\prime}} .
$$

Obviously, there is $P_{n}^{0}=P_{n}$. It is sometimes convenient to use normalized Legendre polynomials $\tilde{P}_{n}$ :

$$
\tilde{P}_{n}(x):=\frac{P_{n}(x)}{\left\|P_{n}\right\|_{L^{2}((-1,1))}}=\sqrt{\frac{2 n+1}{2}} P_{n}(x) .
$$

The following recurrence relations for $P_{n}, \tilde{P}_{n}, P_{n}^{m}$ have been used in Secs. 2 or 4:

$$
\begin{gathered}
(2 n+1) x P_{n}(x)=(n+1) P_{n+1}(x)+n P_{n-1}(x), \\
(2 n+1)\left(1-x^{2}\right) P_{n}^{\prime}(x)=n(n+1)\left(P_{n-1}(x)-P_{n+1}(x)\right), \\
(2 n+1) P_{n}(x)=P_{n+1}^{\prime}(x)-P_{n-1}^{\prime}(x), \\
x \tilde{P}_{n}(x)=\alpha_{n} \tilde{P}_{n+1}(x)+\alpha_{n-1} \tilde{P}_{n-1}(x), \quad \alpha_{n}:=\frac{n+1}{\sqrt{(2 n+1)(2 n+3)}}, \\
\left(1-x^{2}\right)(2 n+1) P_{n}^{\prime m}(x)=(n+1)(n+m) P_{n-1}^{m}(x)-n(n+1-m) P_{n+1}^{m}(x) .
\end{gathered}
$$


The spherical harmonics $Y_{n}^{k}$ with $k \geq 0$ are explained with help of $P_{n}^{m}$ :

$$
Y_{n}^{k}(\theta, \varphi):=(-1)^{k} \sqrt{\frac{2 n+1}{4 \pi}} \sqrt{\frac{(n-k) !}{(n+k) !}} P_{n}^{k}(\cos \theta) e^{i k \varphi}, \quad n \in \mathbb{N}_{0}, \quad k=0, \ldots, n,
$$

and those with $k<0$ are obtained by complex conjugation:

$$
Y_{n}^{-k}(\theta, \varphi):=(-1)^{k} Y_{n}^{k^{*}}(\theta, \varphi), \quad n \in \mathbb{N}_{0}, \quad k=0, \ldots, n .
$$

From (A.2) follows the orthonormality relation

$$
\int_{S^{2}} Y_{n}^{k}(\theta, \varphi) Y_{n^{\prime}}^{k^{\prime *}}(\theta, \varphi) d \Omega=\delta_{n n^{\prime}} \delta_{k k^{\prime}} \quad n, n^{\prime} \in \mathbb{N}_{0}, \quad|k| \leq n,\left|k^{\prime}\right| \leq n^{\prime} .
$$

We note yet the recurrence relation

$$
\sin \theta \frac{\partial Y_{n}^{k}}{\partial \theta}=n N_{n+1}^{k} Y_{n+1}^{k}-(n+1) N_{n}^{k} Y_{n-1}^{k}, \quad N_{n}^{k}:=\sqrt{\frac{n^{2}-k^{2}}{4 n^{2}-1}}
$$

following from (A.8).

B. Appendix. This appendix collects some useful relations concerning the expansion of products of spherical harmonics, resp. of Legendre polynomials (cf. Varshalovich et al. 1988).

Products of spherical harmonics can be expanded in finite sums of spherical harmonics according to

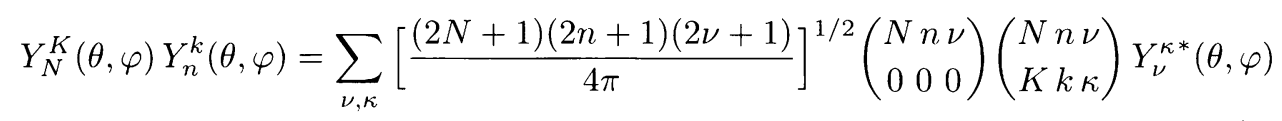

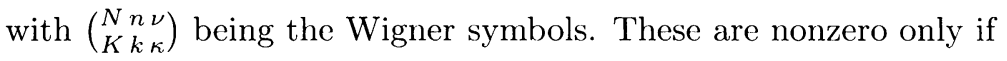

$$
K+k+\kappa=0, \quad|N-n| \leq \nu \leq N+n
$$

moreover, they are real and invariant with respect to cyclic permutations of the rows and obtain a factor $(-1)^{N+n+\nu}$ if two rows are interchanged. They are related to the more commonly used Clebsch-Gordan coefficients $C_{N K n k}^{\nu \kappa}$ by

$$
\left(\begin{array}{l}
N n \nu \\
K k \kappa
\end{array}\right)=(-1)^{\nu+\kappa+2 N} \frac{1}{\sqrt{2 \nu+1}} C_{N-K n-k}^{\nu \kappa} .
$$

Note that the conditions (B.2) ensure the finiteness of the sum in (B.1).

In the axisymmetric situation, (B.1) reduces to

$$
P_{N}(x) P_{n}(x)=\sum_{\nu}(2 \nu+1)\langle N n \nu\rangle P_{\nu}(x),
$$

and for $K=-k=1$ to

$$
P_{N}^{1}(x) P_{n}^{1}(x)=-\sum_{\nu}(2 \nu+1) \frac{1}{2}(\nu(\nu+1)-N(N+1)-n(n+1))\langle N n \nu\rangle P_{\nu}(x),
$$

where we have introduced the abbreviation

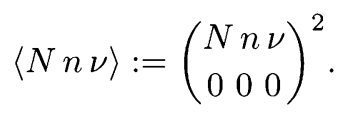


From the properties of the Wigner symbols, it follows that $\langle N n \nu\rangle \geq 0$, the total symmetry and the necessary condition $|N-n| \leq \nu \leq N+n$ for $\langle N n \nu\rangle$ being nontrivial. Moreover, $\langle N n \nu\rangle \neq 0$ implies $N+n+\nu=: 2 p$ with $p \in \mathbb{N}_{0}$. In that case we have the explicit representation

$$
\langle N n \nu\rangle=(-1)^{p}\left[\frac{(p-2 N) !(p-2 n) !(p-2 \nu) !}{(p+1) !}\right]^{1 / 2} \frac{p !}{(p-N) !(p-n) !(p-\nu) !} .
$$

The following recurrence relations follow from the corresponding relations for the Wigner symbols, resp. the Clebsch-Gordan coefficients:

$$
\begin{gathered}
\langle N+1 n \nu\rangle=\frac{(N-n+\nu)(-N+n+\nu+1)}{(-N+n+\nu)(N-n+\nu+1)}\langle N n+1 \nu\rangle, \\
\langle N-1 n \nu\rangle=\frac{(-N+n+\nu)(N-n+\nu+1)}{(N-n+\nu)(-N+n+\nu+1)}\langle N n-1 \nu\rangle, \\
\langle N n+1 \nu\rangle=\frac{\left((N+\nu+1)^{2}-n^{2}\right)\left(n^{2}-(N-\nu)^{2}\right)}{\left((N+\nu+1)^{2}-(n+1)^{2}\right)\left((n+1)^{2}-(N-\nu)^{2}\right)}\langle N n-1 \nu\rangle .
\end{gathered}
$$

Acknowledgements. The authors would like to thank D. Gubbins for pointing to us reference (Hulot et al. 1997) and G. Leugering for reference (Paley \& Wiener 1973). Discussions with J. Bolik, F. Busse, S. Friedrich, M. Krämer, H. Meyer, G. Schneider, C. G. Simader, and W. von Wahl as well as comments from G. Hulot and A. Khokhlov are gratefully acknowledged.

\section{REFERENCES}

Abramowitz, M., Stegun, I. A. (eds): Handbook of mathematical functions, Dover Publications, New York 1972.

Backus, G. E.: Application of a non-linear boundary-value problem for Laplace's equation to gravity and geomagnetic intensity surveys, Quart. J. Mech. Appl. Math. 21, 195-221 (1968).

Backus, G. E.: Non-uniqueness of the external geomagnetic field determined by surface intensity measurements, J. Geophys. Res. 75, 6339-6341 (1970).

Bloxham, J., Jackson, A.: Fluid flow near the surface of earth's outer core, Reviews of Geophysics 29, 1, 97-120 (1991).

Boas, R. P.: Expansions of analytic functions, Transactions of the American Mathematical Society 48, $467-487$ (1940).

Courant, R., Hilbert, D.: Methods of Mathematical Physics, vol II, chapter IV, §1, Interscience Publishers, New York 1962.

Hide, R.: Frozen vector fields and the inverse problem of inferring motions in the electricallyconducting fluid core of a planet from observations of secular changes in its main magnetic field, in: The Physics of the Planets, The Royal Astronomical Society, 1986.

Hulot. G., Khokhlov, A., Le Mouël, J. L.: Uniqueness of mainly dipolar magnetic fields recovered from directional data, Geophys. J. Int. 129, 347-354 (1997).

Kakeya, S.: On the limits of the roots of an algebraic equation with positive coefficients, Tôhoku Math. J. 2, 140-142 (1912).

Kellogg, O. D.: Foundations of Potential Theory, Berlin, Heidelberg, New York 1967.

Kono, M.: Uniqueness problems in the spherical harmonic analysis of the geomagnetic field direction data, J. Geomag. Geoelectr. 28, 11 - 29 (1976).

Merrill, R. T., McElhinny, M. W.: The Earth's Magnetic Field (Its History, Origin and Planetary Perspective), Academic Press, London 1983.

de Sz. Nagy, B.: Expansion Theorems of Paley-Wiener-Type, Duke Math. J. 14, 975-978 (1947).

Paley, R., Wiener, N.: Fourier Transforms in the Complex Domain, The American Mathematical Society, New York 1973. 
Protter, R. P., Weinberger, H. R.: Maximum Principles in Differential Equations, Springer, New York 1984.

Proctor, M. R. E., Gubbins, D.: Analysis of geomagnetic directional data, Geophys. J. Int. 100, 69-77 (1990).

Riordan, J.: Combinatorial Identities, Huntington, New York 1979.

Stoer, J., Bulirsch, R.: Einführung in die Numerische Mathematik II, 2nd ed., Berlin, Heidelberg, New York 1978.

Varshalovich, D. A., Moskalev, A. N., Khersonskii, V. K.: Quantum Theory of Angular Momentum, World Scientific, Singapore, New Jersey, Hong Kong 1988. 